\title{
Molecular Dynamics and Metadynamics Insights of 1,4- Dioxane Induced Structural Changes of Biomembrane Models
}

Zsófia Borbála Rózsa, Lukács József Németh, Balazs Jojart, Károly Nehéz, Béla Viskolcz, and Milán Sz\#ri J. Phys. Chem. B, Just Accepted Manuscript • DOI: 10.1021/acs.jpcb.9b04313 • Publication Date (Web): 27 Aug 2019

Downloaded from pubs.acs.org on August 27, 2019

\section{Just Accepted}

"Just Accepted" manuscripts have been peer-reviewed and accepted for publication. They are posted online prior to technical editing, formatting for publication and author proofing. The American Chemical Society provides "Just Accepted" as a service to the research community to expedite the dissemination of scientific material as soon as possible after acceptance. "Just Accepted" manuscripts appear in full in PDF format accompanied by an HTML abstract. "Just Accepted" manuscripts have been fully peer reviewed, but should not be considered the official version of record. They are citable by the Digital Object Identifier (DOI®). "Just Accepted" is an optional service offered to authors. Therefore, the "Just Accepted" Web site may not include all articles that will be published in the journal. After a manuscript is technically edited and formatted, it will be removed from the "Just Accepted" Web site and published as an ASAP article. Note that technical editing may introduce minor changes to the manuscript text and/or graphics which could affect content, and all legal disclaimers and ethical guidelines that apply to the journal pertain. ACS cannot be held responsible for errors or consequences arising from the use of information contained in these "Just Accepted" manuscripts. 


\title{
Molecular Dynamics and Metadynamics Insights of 1,4- Dioxane Induced Structural Changes of Biomembrane Models
}

\author{
Zsófia Borbála Rózsa ${ }^{a}$, Lukács József Németh ${ }^{b}$, Balázs Jójárt ${ }^{b}$, Károly \\ Nehéz ${ }^{c}$, Béla Viskolcz ${ }^{\mathrm{a}}$, Milán Szőria*
}

a Institute of Chemistry, University of Miskolc, Egyetemváros A/2, H-3515 Miskolc, Hungary

${ }^{b}$ Department of Food Engineering, Faculty of Engineering, University of Szeged, Mars tér 7. 6724, Szeged, Hungary

${ }^{c}$ Department of Information Engineering, University of Miskolc, MiskolcEgyetemváros Informatics Building, H-3515 Miskolc, Hungary

*e-mail: milan.szori@uni-miskolc.hu 


\begin{abstract}
1,4-dioxane is a cytotoxic B2 type human carcinogen, a serious water pollutant produced solely by industrial activity. The effect of 1,4-dioxane on phospholipid membrane models composed by DPPC and its branched isomer (IPPC) was investigated using MD simulations. Clear and polluted membranes were compared by membrane parameters such as APL, VPL, compressibility modulus, membrane thickness and orderliness of lipid tails. While neat systems significantly differ from each other, the presence of the pollutant has the same effect on both types of lipid membranes: high density of dioxane appears at the vicinity of ester groups which pushes away lipid headgroups from each other, leading to an overall change in lipid structure: APL and VPL grows, while the orderliness of lipid tails, membrane thickness and compressibility modulus decreases. Orientational preferences of water and dioxane molecules were also investigated and different membrane regions have been specified according to the stance of water molecules. Free energy profile for 1,4-dioxane penetration mechanism into DPPC membranes was carried out using metadynamics for two different concentrations of the pollutant $\left(c_{1}=7.51 \mathrm{~g} / \mathrm{dm}^{3}, c_{2}=75.10 \mathrm{~g} / \mathrm{dm}^{3}\right)$, which showed that the higher the concentration is, the lower the free energy of penetration gets. Only a small free energy barrier was found in the headgroup region and accumulation of dioxane is thermodynamically unfavored in the middle of the bilayer. The penetration mechanism has been described in detail based on the orientational preference of 1,4-dioxane molecules and the free energy profiles.
\end{abstract}




\section{Introduction}

The passive membrane permeation of small molecules plays important role in their disposition and toxicity ${ }^{1}$. Passive diffusion is the main limitation of penetration of small molecules and thus the barrier for their infiltration into living cells (human, animal and bacteria). A key property of the passive transport of an investigated substance is its partitioning between water and membrane phases which is affected by the kinetics and thermodynamics of the specific permeation process ${ }^{2,3,4}$.

In most mammalian and bacterial cells phospholipids compose the base of the lipid bilayer with different types of head groups. In eukaryotic cells phosphatidylcholine (PC) is the most commonly found headgroup type for phospholipids $(\sim 50 \%)$, but others, like phosphatidylserine phosphatidylethanolamine, sphingomyelins and cholesterol can also be found as membrane components ${ }^{5}$. In the case of prokaryotic cells it is estimated that only $10 \%$ of bacteria produces phosphatidylcholines, while phosphatidylglycerols and phosphatidylethanolamines are the most common lipid types ${ }^{6}$. Thus both experimental and theoretical models usually utilize simple phospholipid bilayers to understand solute permeation processes ${ }^{2}$.

In terms of lipid chains several different types can be observed in cells, which can be characterized by their length, degree of unsaturation and branching. Branching fatty acids are widespread mostly among bacterial cell membranes. The most common ones contain one methyl group at the iso or anteiso position of the fatty acid ${ }^{7,8}$. In general both poly- and monomethylbranching is associated with increasing the area per lipid, while decreasing the bilayer thickness and lowering chain ordering, thus enhancing the fluidity of the plasma membrane ${ }^{8,9}$. It has great importance in case of archaea and bacteria living in extreme conditions ${ }^{8}$. Iso- and midchain methylbranching is associated with increasing the tolerance of the cell membrane to toxic compounds ${ }^{10}$. In the case of polymethyl-branching fatty acids - like phytanyl and phitanoyl - containing membranes it has been suggested, that they enhance the structural stability of cell membranes thus making it less permeable to water molecules, ions and small solutes compared to cell membranes composed of only straight chained lipids ${ }^{11}$, but through computational studies it has been proven that branching has no significant effect on the partition coefficients of neutral solutes ${ }^{12}$. These molecules have also been associated with the disturbance of the membrane interior's cavity distribution ${ }^{12}$ which leads to a reduced amount of water molecules diffusing inside the membrane, while their mobility is also reduced.

Membrane/water interfaces are critical to many cellular functions. Water influences stability, fluidity and phase behavior of phospholipid membranes, and also has a crucial role in the electric properties of the bilayer ${ }^{13,14}$. Both experiments and molecular simulations have shown that water molecules lose their bulk-like nature near membrane surfaces 15,16,17,18. Translational mobility and rotational entropy of individual water molecules decrease when their interactions with the lipid 
headgroups become stronger ${ }^{19}$ resulting in different interfacial water regions according to the membrane composition ${ }^{13,20}$. The total membrane dipole potential is a manifestation of the nonrandom orientation of the electric dipoles of headgroups, carbonyl groups and water ${ }^{14,15}$. The hydrating water molecules form an ordered, typically 1-2.5 nm thick stable layer (maximum 5 layers of structured water) at the membrane interface ${ }^{13}$, where adjacent water molecules are oriented by the headgroups as the dipoles of individual water molecules are aligned against the bilayer dipole ${ }^{14}$. The ester groups on the internal side of the headgroups can also establish hydrogen bonds closest to the bilayer center, which helps to create the dense, ordered state at the region of the acyl chains of the phospholipids, thus creating a barrier of penetration ${ }^{14}$. These layers are stable enough to present multiple energy barriers to approaching nanoscale objects ${ }^{16,21}$. Preferred molecular orientations can capture most of the structural features of these interfacial layers and they can be used for defining interfacial sublayers. The orientation of molecules relative to a membrane/water interface can be analyzed in terms of the orientation statistics of various vectors bound to the target molecule $22,23,24$.

Due to the complex nature and structure of biomembranes, its sublayers can be split into regions with different characteristics. There are several ways to split up the bilayer based on the investigated phenomena ${ }^{25,26}$ from which we hereby adapted the four region model proposed by Marrink and Berendsen ${ }^{27}$ :

Region I: The outer region of lipid bilayers, where the orientation of water molecules is already perturbed due to the headgroups and thus their diffusion constants, molecular orientations and solubilities differ from the aqueous bulk. Its border can be defined where lipid and water densities become comparable.

Region II: Highly structured region with a high mobile charge density of the head groups which can be a limiting factor for the penetration of polar and charged molecules. According to the original definition this region lasts until water density drops below $1 \%$.

Region III: A region with high lipid chain density. It is considered as the main barrier for the permeation process of small molecules, as hydrophilic penetrants are not soluble in this region and water density is below $1 \%{ }^{28}$.

Region IV: The middle of the bilayer where terminal methyl groups are found in the presence of a high fraction of free volume, therefore it is also called a low acyl chains density region.

Molecular Dynamics (MD) is a powerful tool to describe and understand the time evolution of a computational model of a complex chemical (biochemical) system. In the case of classical MD simulations the interatomic interactions are described by force fields - which are mathematical expressions describing the dependence of the systems potential energy on the atomic positions -, and atomic motions with the Newtonian equations of motion ${ }^{29}$. MD enables a thorough view of structure and dynamics of the simulated system and can provide relevant details which would be either 
inaccessible or expensive and time consuming via experiments ${ }^{30}$. In the case of biological membranes several studies have been carried out recently with the help of MD to understand - for example - the structure and phase transition of membrane models of different lipid compositions 31,32 ; the selfassembly of bilayers ${ }^{33,34}$; the effect of ions ${ }^{35}$; interactions with proteins ${ }^{36,37}$; the permeation of small molecules $3,38,39,40,41$.

While the structural changes of such processes can be understood via the analysis of general membrane parameters ${ }^{42,43}$, the energetic properties of the interactions remain hidden. The partitioning and preferred position of an interacting molecule - like in the case of membrane penetration - can be determined by a free energy profile along the bilayer normal where the change in the free energy provides a clear view whether a process is thermodynamically favorable or not. Thus free energy calculations are an efficient tool to estimate kinetic and dynamic characteristics of chemical and biological processes, like rate constants, permeability coefficients or ionic conductance 44,45. To obtain the free energy profile of such process classical MD simulations are not well suited, as high free energy barriers restrict the detailed sampling of the entire configuration space as the system is likely to be found trapped in local free energy minima. Thus enhanced sampling algorithms were developed which bias the system towards high energy regions which would be inaccessible otherwise ${ }^{38,46}$ such as umbrella sampling ${ }^{47}$, adaptive biasing ${ }^{48}$, thermodynamic integration ${ }^{49}$, metadynamics ${ }^{50,51}$ and its improved version, well-tempered metadynamics ${ }^{52}$.

1,4-dioxane $\left(\mathrm{C}_{4} \mathrm{H}_{8} \mathrm{O}_{2}, \mathrm{M}_{\text {DIOX }}=88.11 \mathrm{~g} / \mathrm{mol}\right)$ is a pleasant odour synthetic industrial chemical with no known natural sources. Before the Montreal Convention it has been used as a stabilizing agent for chlorinated solvents, primarily during the production of 1,1,1-trichloroethane (TCA). Nowadays, it is a widely used industrial solvent during the production of organic compounds, disinfectants, cooling liquids and hygienic products. It also appears as a side product of several industrial processes, like PET manufacturing 53,54. 1,4-dioxane pollution has been detected all over the world in different areas, from surface water, groundwater to landfills, mostly coming from industrial wastewater, industrial waste and illegal dumping 54,55,56.

At the same time 1,4-dioxane has several health effects. Inside the body it is instantly adsorbed through the lungs and the gastrointestinal tract, and distributes quickly and uniformly between the lungs, liver, kidney, spleen, colon and skeletal muscle tissues. Animal studies proved ${ }^{54}$ that it causes kidney and liver cancer, thus it is considered as a B2 type 'Probable human carcinogen' 57. 1,4dioxane appears to be a typical cytotoxic carcinogen, but it requires a high level of exposure for its metabolism to happen ${ }^{58}$. As the substance has nonlinear toxicokinetics it can accumulate in the body, mostly in the liver. In rats it has been shown that its half-life increases with the dose of 1,4-dioxane from $1 \mathrm{~h}$ to $14 \mathrm{~h}{ }^{59}$. 1,4-dioxane eliminates the body after its metabolism into 2-(2hydroxyethoxy)acetic acid (HEAA) via urination, of which the amount depends on the consumed 
dose ${ }^{60}$.

The physico-chemical properties of 1,4-dioxane make it a dangerous and persistent groundwater pollutant, as it is almost completely miscible with water ${ }^{61}$, its density is roughly the same as water $(1.0329 \mathrm{~g} / \mathrm{mL})^{62}$ and its low octanol-water partition coefficient $\left(\log \mathrm{K}_{\mathrm{OW}}=-0.27\right){ }^{63,64}$ indicating that it is also compatible with lipophilic matrices.

Despite the amphiphilic nature of 1,4-dioxane and its serious health risk, to the best of our knowledge, neither its passive membrane permeation nor its effect on the membrane structure was not investigated yet. Therefore, the aim of our study was to get detailed information about structural changes in biomembranes caused by 1,4-dioxane using DPPC (dipalmitoyl-phosphatidylcholine) and IPPC (isodipalmitoyl-phosphatidylcholine, 1-14-methylpentadecanoyl-2-palmitoylphosphatidylcholine, 14-MpPPC, Figure 1) model membrane systems. DPPC membranes are one of the most extensively investigated model bilayer systems ${ }^{65,28}$ and IPPC only differs in a branching methyl group at the $\mathrm{C} 14$ position of one of the hexadecanoates ${ }^{9}$. Changes induced by 1,4-dioxane are analyzed through the inflicted alterations in general membrane parameters, density profiles, area per lipid (APL), volume per lipid (VPL), isothermal compressibility $\left(\mathrm{K}_{\mathrm{A}}\right)$, deuterium order parameter $\left(\mathrm{S}_{\mathrm{CD}}\right)$ and membrane thickness $\left(\mathrm{D}_{\mathrm{HH}}\right)$. To thoroughly describe the penetration mechanism orientations of water and the pollutant molecules in the membrane/water interfacial regions were described using vectors bound to the molecules. Furthermore, we also determined the concentration dependence of the height of the free energy barrier of 1,4-dioxane penetration in order to estimate how easily it can cross biomembranes to exert its carcinogenic effects. 


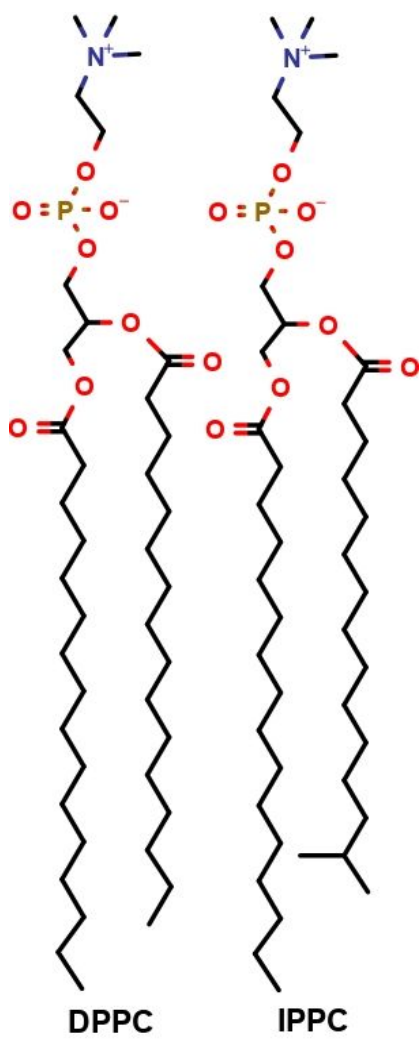

Figure $12 D$ structure of DPPC and IPPC molecules 


\section{Methods}

\section{System Preparation}

Total four different systems were used to understand the effects of 1,4-dioxane: DPPC and IPPC model membranes, and DPPC and IPPC membranes (Figure 1) in the presence of the pollutant molecule. Throughout this work pure systems will be referred to as 'clear', and 1,4-dioxane containing systems as 'polluted' systems. Initial membrane configurations have been generated using automated online membrane builder, CHARMM-GUI ${ }^{66,67}$. The simulated systems consisted of $128\left(n_{\text {lipid }}=128\right.$, 64-64 lipid molecules each membrane leaflet, which size have been proven to be sufficiently large to reproduce membrane structural parameters ${ }^{3,38,41,68,69}$ ) either DPPC or IPPC molecules, with 50 water molecules per headgroup ( $n_{\text {water }}=6400$ in total), which is well above the value of the water molecules needed for total hydration of bilayer ${ }^{70}$. In the case of polluted systems an additional 100 1,4-dioxane molecules have been inserted into the simulation box in random positions. Such high concentration $\left(75.102 \mathrm{~g} / \mathrm{dm}^{3}\right)$ had been chosen to exaggerate studied effects to distinguish it from statistical noise as suggested by $\mathrm{Oh}$ and Klein ${ }^{71}$. Lipid molecules were modelled by CHARMM36 ${ }^{72}$, water molecules by CHARMM TIP3P ${ }^{73}$ and CGenFF ${ }^{74}$ force field parameters were applied for 1,4-dioxane molecules in a cubic simulation box using periodic boundary conditions. All dynamics calculations were carried out using GROMACS 5.1.2 ${ }^{75}$, while VMD ${ }^{76}$ was used for molecular visualization.

\section{Parameters of MD simulations}

Production runs have been set up with an equilibration protocol where the number of optimization steps has been maximized at 5000 . This was followed by a 25 ps long simulation using NVT ensemble where at first the $P$ atoms were restrained to their positions with a $1000 \mathrm{~kJ} /\left(\mathrm{mol} \cdot \mathrm{nm}^{2}\right)$ force constant along axis $z$ to prevent decomposition of the bilayer ${ }^{72}$. The next equilibration step was 800 ps long in an $\mathrm{Np}_{\text {semi-isotropic }} \mathrm{T}$ ensemble. The temperature $(\mathrm{T}=330 \mathrm{~K}$, above the phase transition temperature, which is $313.35-313.45 \mathrm{~K}$ experimentally $\left.{ }^{77,78,79}\right)$ and pressure $(\mathrm{p}=1 \mathrm{~atm})$ was set using Berendsen temperature and pressure control ${ }^{80}$, where the value of coupling constants were chosen as $\tau_{\mathrm{T}}=1.0 \mathrm{ps}$ and $\tau_{\mathrm{p}}=5.0 \mathrm{ps}$. Non-bonded interactions have been calculated using $1.2 \mathrm{~nm}$ cut-off value ${ }^{72}$, while the long distance electrostatic interactions have been accounted using smooth Particle Mesh Ewald summation method ${ }^{81}$. During calculations the heavy-atom-hydrogen distances have been fixed by the means of P-LINCS algorithm ${ }^{82}$ with the exception of water molecules which have been kept rigid by using SETTLE ${ }^{83}$. The equations of motion were integrated in 2 fs time steps and structures have been saved to a trajectory file every $10 \mathrm{ps}$. Further simulations have been carried out in $\mathrm{Np}_{\text {semi- }}$ isotropic $T$ ensemble using Nosé-Hoover temperature ${ }^{84,85}$ and Parrinello-Rahman pressure ${ }^{86}$ control for 125 ns. Temperature and pressure values were the same as during previous $\mathrm{Np}_{\text {semi-isotropic }} \mathrm{T}$ equilibration to maintain a biologically relevant liquid-crystalline $\left(\mathrm{L}_{\alpha}\right)$ phase. Out of these simulations 
the first $25 \mathrm{~ns}$ were considered as part of the equilibration protocol, as the box sizes were still drastically changing in the case of 1,4-dioxane polluted systems, thus it was left out from the analysis, and the remaining $100 \mathrm{~ns}$ was considered as the production run. For each system 5 parallel production runs were conducted, resulting in $500 \mathrm{~ns}$ of total simulation for clear and polluted systems.

\section{Analysis of structural membrane parameters}

The analysis of membrane parameters was carried out using the equations listed in Table 1. Membrane thickness was calculated as the distance between the two peaks of the electron density profiles of the systems ${ }^{87}$.

Table 1 Definition of the used membrane parameters.

\begin{tabular}{|c|c|c|c|}
\hline $\begin{array}{l}\text { Membrane } \\
\text { parameter }\end{array}$ & Definition & Formula & Abbreviation \\
\hline APL & Area per lipid ${ }^{88}$ & $\frac{2 \times L_{x} \times L_{y}}{n_{\text {lipid }}}$ & $\begin{array}{l}L: \text { average } \mathrm{x} \text { and } \mathrm{y} \\
\text { dimensions of the } \\
\text { simulation box }\end{array}$ \\
\hline VPL & Volume per lipid ${ }^{88}$ & $\frac{V_{\text {box }}-n_{\text {water }} V_{\text {water }}-n_{\text {dioxane }} V_{\text {dioxane }}}{n_{\text {lipid }}}$ & $\begin{array}{c}V_{b o x}: \text { average volume } \\
\text { of the simulation box } \\
V_{\text {water }} \text { : the volume of a } \\
\text { TIP3P water molecule } \\
\left(0.03053 \mathrm{~nm}^{3}\right)^{87} \\
V_{\text {dioxane }}: \text { derived from } \\
\text { the density of neat } 1,4 \\
\text { dioxane }\left(1.03 \mathrm{~g} / \mathrm{cm}^{3} 5\right. \\
\left.\quad-0.146 \mathrm{~nm}^{3}\right)\end{array}$ \\
\hline $\mathbf{K}_{\mathbf{A}}$ & $\begin{array}{l}\text { Isothermal } \\
\text { compressibility modulus, } \\
\text { a dynamic parameter to } \\
\text { measure stress resistance } \\
\text { of the membrane } \\
89\end{array}$ & $\frac{2 k_{B} T\langle A P L\rangle}{n_{\text {lipid }} \sigma_{A P L}^{2}}$ & $\begin{array}{c}k_{b}: \text { Boltzmann's } \\
\text { constant } \\
T: \text { temperature } \\
\sigma_{A P L}: \text { the variance of } \\
\text { APL }\end{array}$ \\
\hline $\mathbf{S}_{\mathbf{C D}}$ & $\begin{array}{l}\text { Deuterium order } \\
\text { parameter, which } \\
\text { measures the orientation } \\
\text { of C-H bonds with } \\
\text { respect to the bilayer } \\
\text { normal }{ }^{87}\end{array}$ & $\frac{1}{2}\left\langle 3 \cos ^{2} \Theta-1\right\rangle$ & $\begin{array}{c}\Theta: \text { the angle between } \\
\text { bilayer normal }(\mathrm{Z}) \text { and } \\
\text { the vector between } \\
\mathrm{C}_{\mathrm{i}}-\mathrm{H}_{\mathrm{i}}\end{array}$ \\
\hline
\end{tabular}

\section{Definition of vectors bound to water and 1,4-dioxane molecules}

To be able to describe the orientational preference of water and 1,4-dioxane molecules in the membrane/water interface vectors were defined bound to each investigated species. Three local vectors have been defined to describe the orientation of water molecules (upper panel of Figure 2): the dipole vector $(z)$, the normal vector $(x)$ and the vector joining the two hydrogen atoms (H-H vector, $y$ ) in order to obtain their relative orientation to the membrane surface normal $(Z)$. Their orientation is characterized by their angles denoted $\alpha, \beta$ and $\gamma$ respectively, formed with the interface normal. In 
the case of 1,4-dioxane two vectors and the corresponding angles $(\alpha, \beta$ in the bottom panel of Figure 2) have been chosen, the vector joining the two oxygen atoms (O-O vector, $z$ ), and the normal of this vector $(x)$. It should be noted that due to the symmetry of the molecules the molecular normal, and $\mathrm{H}-\mathrm{H}$ vectors can be directed in two opposite directions, and there is no physical way to distinguish them. Therefore, their corresponding angles ( $\beta$ and $\gamma$ ) can only be scattered between $0^{\circ}$ and $90^{\circ}$, and thus their cosines between 0 and 1, while $\alpha$ angles defined by the dipole moment vector of water or $\mathrm{O}-\mathrm{O}$ vector of dioxane (as a direction is defined by selecting the $\mathrm{O}-\mathrm{O}$ vector) can fall into the entire angular range of $0^{\circ}$ to $180^{\circ}$, and cosine range of -1 and 1 . To make interpretation easy for their ensemble averages, uniformly distributed orientation belongs to value 0 , which is the case for the expected value $(\langle\rangle)$, of $\cos (\alpha)$, while $\cos (\beta)$ and $\cos (\gamma)$ must be shifted downwards by 0.5 . To characterize the variations of the average alignment of water and dioxane molecules along the interface normal axis, $Z$ has been divided into $1 \AA$ slices, where $\Phi_{\alpha}(Z), \Phi_{\beta}(Z)$ and $\Phi_{\gamma}(Z)$ - orientational function - profiles have been calculated, defined as:

$$
\begin{array}{ll}
\Phi_{\alpha}(Z)=\rho(Z)\langle\cos (\alpha)\rangle(Z) & \text { Eq. } 1 \\
\Phi_{\beta}(Z)=\rho(Z)\langle\cos (\beta)-0.5\rangle(Z) & \text { Eq. } 2 \\
\Phi_{\gamma}(Z)=\rho(Z)\langle\cos (\gamma)-0.5\rangle(Z) & \text { Eq. } 3
\end{array}
$$

where $\rho(Z)$ is the number density of the molecule (water or dioxane) in the corresponding slice of the simulation box. It is important to note that while any two of these angles can describe the orientation of a single molecule relative to the interface, these angles are not independent of each other. To create bivariate angle distribution maps of the water and 1,4-dioxane molecules two independent angles have been chosen as angular polar coordinates of the interface normal vector (Figure 2): $\alpha$ and $\delta$, which are the angle of the molecular dipole vector enclosing with the bilayer normal, and the angle formed by the molecular normal vector and the projection of the interface normal $(Z)$ to the plane perpendicular to the molecular dipole vector ( $x y$ plane, shown in blue in Figure 2), respectively. For local Cartesian coordinates were fixed to each investigated molecule, where $x$-axis corresponds to the molecular normal, $y$ to the $\mathrm{H}-\mathrm{H}$ and $z$ to the molecular dipole vector (Figure 2). It should be noted, that angle $\delta$ can always be chosen in a way to fall in the range of $0^{\circ} \leq \delta \leq 90^{\circ}$ because the normal can point towards two opposite directions. 

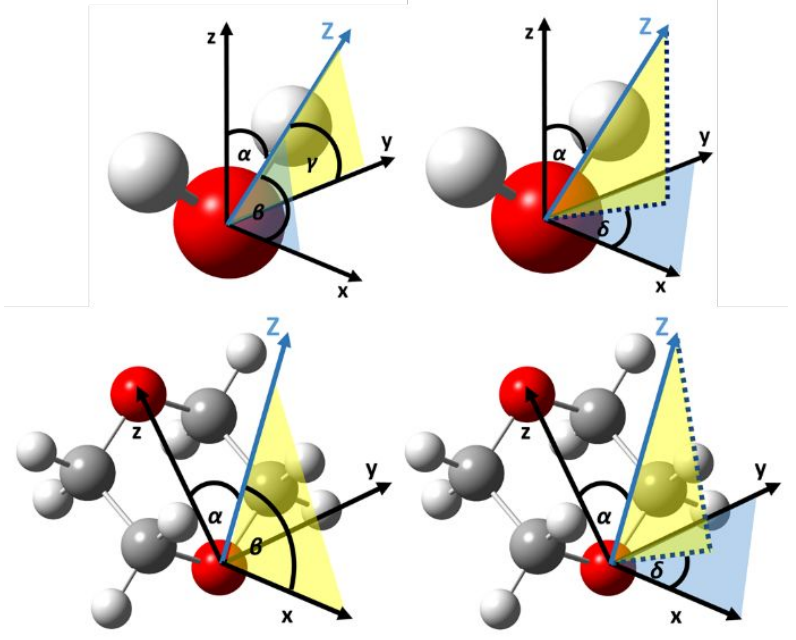

Figure 2 Definition of the local vectors $(x, y, z)$ bound to water and 1,4-dioxane molecules. The orientation of the molecules relative to the membrane normal $(Z)$ are defined by $\alpha, \beta, \gamma, \delta$.

\section{Free energy calculations}

In metadynamics 50,52 , a history-dependent bias potential $(P(s, t))$ is calculated along a collective variable $(s)$, which itself is a function of the positions of the particles throughout the simulation time $(t)$. The bias potential appears during the simulations as a repulsive Gaussian, centered on the explored points of a collective variable space $(C V(s))$, leading the system towards previously unexplored configurations. These Gaussians have a preassigned width $(\sigma)$ and height $(\omega)$ and are deposited at every time step $\left(t^{\prime}\right)$ as the simulation proceeds, where the collective variable has a value of $s\left(t^{\prime}\right)$. Then the bias potential can be calculated the following way:

$$
P(s, t)=\omega_{0} \sum_{t^{\prime}<t} \mathrm{e}^{\frac{\left(s-s\left(t^{\prime}\right)\right)^{2}}{2 \sigma^{2}}}
$$

By adding these Gaussians the system is discouraged of the exploration of already visited states of $\mathrm{CV}(\mathrm{s})$, and their sum provides an immediate estimate of the free-energy surface $(\mathrm{F}(\mathrm{s}))^{50}$.

While metadynamics has been used successfully in a large variety of membrane penetration studies of different small molecules $90,91,92,93,94$ it still has problems arising from that the free energy not converges to an exact value but fluctuates around the correct result as similar sized Gaussians are deposited along the $\mathrm{CV}{ }^{52,38}$. Thus well-tempered metadynamics (WT-MD) ${ }^{52}$ was proposed, where the height of the hills decreases as passing by previously mapped regions of the free energy profile which enables the convergence of the profile to an exact value ${ }^{95,46}$. The rescaling of the height of the Gaussians $\left(\omega_{t^{\prime}}\right)$ goes the following:

$$
\omega_{t^{\prime}}=\omega_{0} e^{-\frac{P\left(s, t^{\prime}\right)}{k_{B} \Delta T}},
$$

where $\Delta \mathrm{T}$ is an input parameter in units of temperature and $\mathrm{k}_{\mathrm{B}}$ is the Boltzmann constant. Than if we 
replace $\omega_{0}$ to $\omega_{t}$, in Eq 4, the bias potential on a long time limit converges to:

$$
P(s, t \rightarrow \infty)=-\frac{T+\Delta T}{\Delta T} F(s)+C, \quad \text { Eq. } 6
$$

where $C$ is an arbitrary constant and $\frac{T+\Delta T}{\Delta T}$ is the bias factor in which $\mathrm{T}$ is the simulation temperature.

To characterize the energetic nature of the penetration mechanism of 1,4-dioxane, metadynamics simulation protocols have been carried out using PLUMED 2.3.0 96,97 combined with GROMACS 5.1.4 ${ }^{75}$. Here, Gaussians were deposited at every $2500 \mathrm{ps}$, with a height of $0.1 \mathrm{~kJ} / \mathrm{mol}$ and width of $0.5 \AA$. Walls for the pulling were set $37 \AA$ from the center of the membrane bilayer. The 1000 ns simulation time was analyzed while other parameters of the metadynamics were identical to the production runs. The free energy profile of the penetration can depend on the concentration of the dioxane, therefore an additional 500 ns long metadynamics simulation had also been carried out for a system having an order of magnitude less dioxane in the simulation box (10 dioxane molecules).

As mentioned before, metadynamics simulations can suffer from convergence issues, thus an additional 600 ns well-tempered metadynamics simulation has been run for the higher pollutant concentration, to have a guess whether the penetration process has a method dependence. During the WT-MD calculations Gaussians were deposited at every $3000 \mathrm{ps}$, with a height of $2.2 \mathrm{~kJ} / \mathrm{mol}$ and width of $0.1 \AA$, while the bias potential was set to be 6.0. Other parameters of the WT-MD were identical to metadynamics calculations.

\section{Results and Discussion}

Density profiles

The symmetrized and centered mass density profiles along the membrane normal are shown in Figure 3 for the four studied systems. Besides the total mass density, the spatial contributions of water molecules and 1,4-dioxane as well as that of choline, phosphate, ester and methyl groups of DPPC/IPPC are presented. The obtained electron density profiles of clear DPPC bilayer agrees reasonably well with experimental data 70 and the comparison can be found in the SFigure 1 of Supplementary Information. The clear DPPC and IPPC density profiles differ only in the density for the terminal methyl groups in the middle of the bilayer (at $Z \approx 0 \AA$ ) due to the branching of the isopalmitic-acid in IPPC membrane. Presence of 1,4-dioxane as a pollutant affects both membranes similarly. The mass density profile of the pollutant indicates a local maximum at the inner side of the ester groups (at $|Z| \approx 12 \AA$, which is the border of Region III and IV). Here, dioxane density ( $\rho_{\text {DIOX, }}$ ester $\left.=0.042 \mathrm{~g} / \mathrm{cm}^{3}\right)$ is more than half of its aqueous phase density $\left(\rho_{\text {DIOX, bulk }}=0.068 \mathrm{~g} / \mathrm{cm}^{3}\right)$, while it is significantly smaller for the remaining part of the membrane. In the inner regions of the membrane a small accumulation can be observed around $5 \AA$ distance of the penetrating 1,4-dioxane molecules radial distribution function which can be found in the SFigure 2 of Supplementary Information. 
Furthermore, as an effect of dioxane pollution a slight growth in water density can also be observed at $|Z| \approx 17 \AA$ which means more water molecules can access the high density of acyl chains region (Region III) of the membranes.
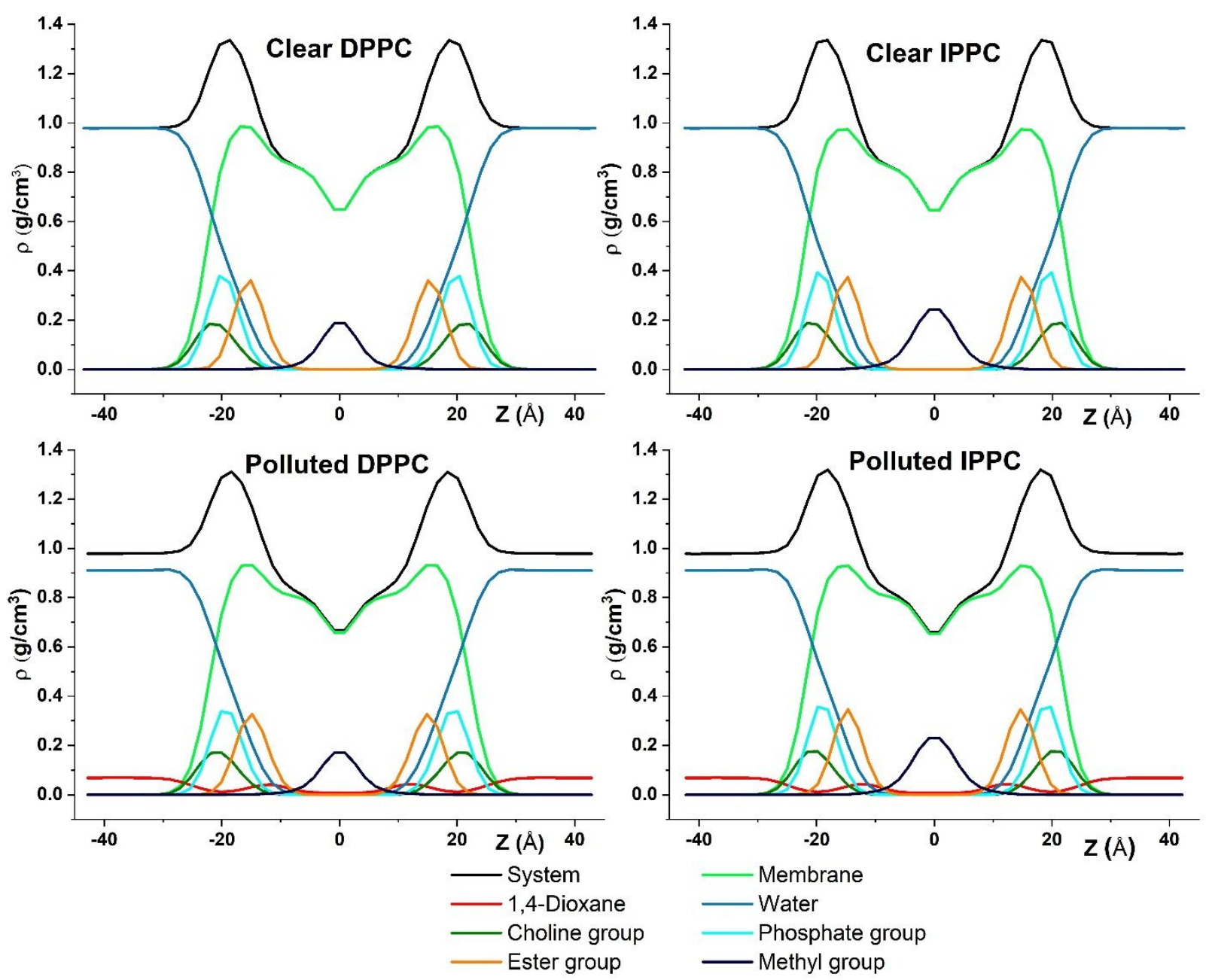

Figure 3 Mass density profiles of the membrane systems along the membrane normal $(Z)$. The overall mass densities are decomposed into subsections to highlight the inner structure of the biomembranes. Black - complete system, green DPPC/IPPC, blue - water, dark green - cholines, cyan - phosphates, orange - esters, dark green - terminal methyl groups, red-1,4-dioxane.

\section{Area per Lipid}

Calculation of area per lipid (APL) is one of the most generally applied test for membrane simulations ${ }^{98}$ since APL is accessible experimentally via NMR $^{99}$, neutron diffraction ${ }^{100}$ or X-ray 101 measurements. It is worth mentioning that literature APL values are scattered in a large range depending on its indirect measurement and state of the membrane phase ${ }^{98}$. Based on molecular simulations, the average APL can also be derived from different methods. Conventionally, it is calculated from the lateral dimensions of the simulation box divided by the number of lipids in a membrane leaflet (Table 1), but in this case the APL distribution remains unknown. Besides the 
conventional APL calculation, the average APL can also be obtained via a Voronoi tessellation (VT) based technique for simple and multicomponent lipid bilayers ${ }^{102,103}$ as well as complex proteinmembrane ${ }^{104,105}$ systems. The great advantage of VT technique is that it also provides not only APL distribution beyond the average but also local structural information of the bilayer surface ${ }^{105}$. For instance, local disturbances caused by the direct permeation of small molecules can be identified by VT-based APL distribution 106,107,102. To find such local disturbances, the NpT trajectories had been analyzed by a VT-based in-house Python script (powered by Python 2.7108 and MDAnalysis 0.18.1 ${ }^{109}$ ), in which the phosphorous atom was projected to the macroscopic $X Y$ surface of the membrane ${ }^{110}$, while the periodic boundary conditions were also taken into account. The area belonging to each phospholipid molecule has been calculated as the area of a Voronoi polygon by using Delaunay triangulation ${ }^{111}$. The VT-based APL calculation has been carried out for all 5 runs of the individual systems to obtain statistically relevant results. The comparison of the different types of average APL calculations and the distributions of the VT-based calculations are shown in Figure 4 and Figure 5, respectively.

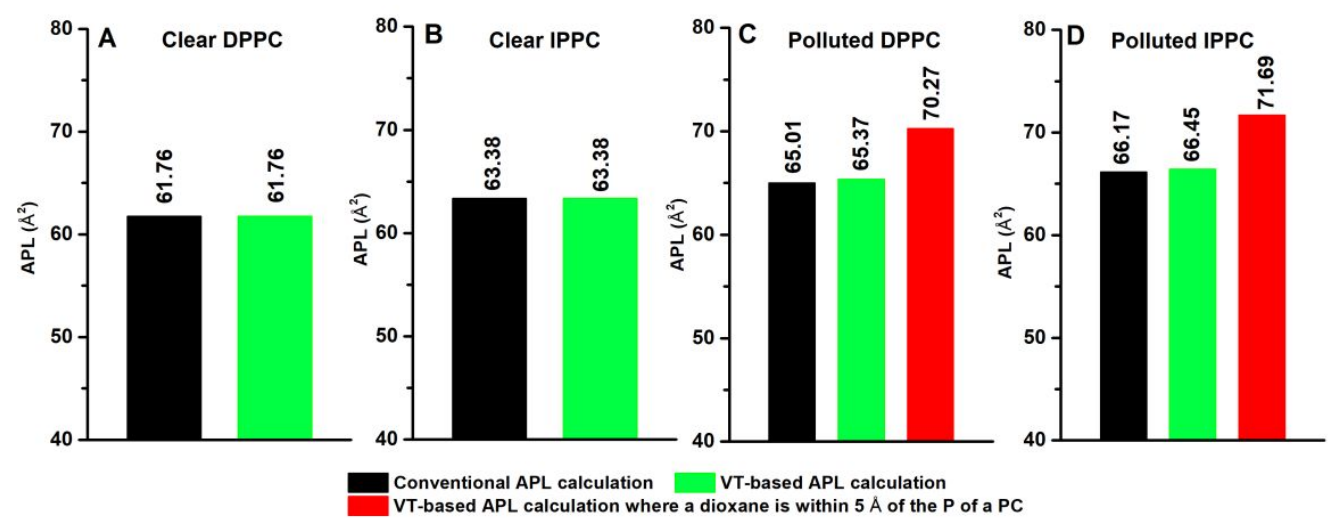

Figure 4 Average area per lipid (APL) calculated by conventional and Voronoi tessellation-based approaches for DPPC and IPPC membranes without (the first two diagrams) and with the presence of 1,4-dioxane (the last two diagrams). Average APL obtained from all phosphorus atoms using conventional (black) and Voronoi tessellation approach (green). APL is also calculated from all phosphorus atoms which have 1,4-dioxane molecules within its $5 \AA$ radius (red). 


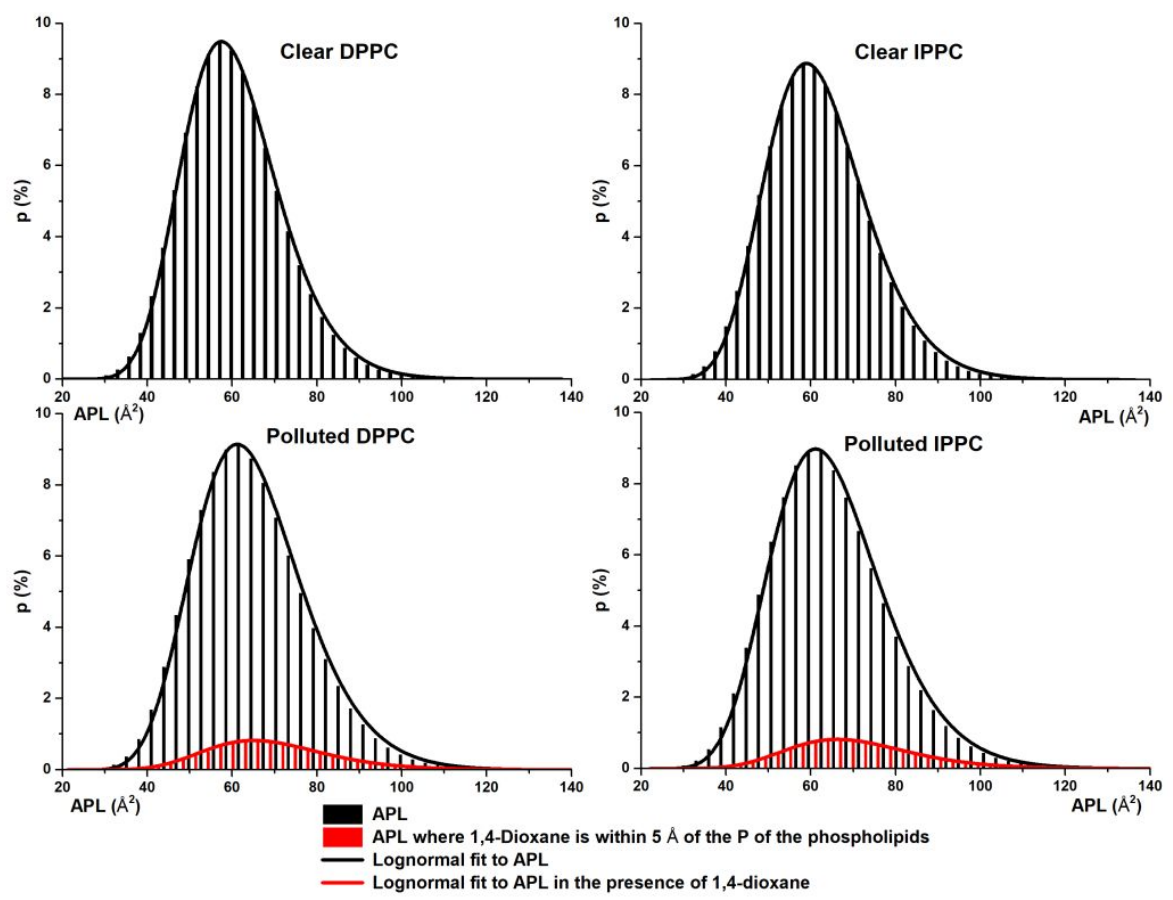

Figure 5 Probability distributions of area per lipid (APL) obtained by Voronoi tessellation approach. The total APL distribution isshown in black while the local APL distribution of phosphorus atoms which have 1,4-dioxane molecules within its $5 \AA$ radius are in red. Lognormal distribution fits are also indicated in the diagrams.

In the case of the clear systems, the average APL values obtained from conventional and VTbased approaches match perfectly (see the first two panels of Figure 4) and their relative deviation from experiment is only $2 \%$ in the case of DPPC $\left(\mathrm{APL}_{\text {calc }}=61.76 \pm 1.44 \AA^{2}, \mathrm{APL}_{\text {exp }}=62.9 \pm 1.3 \AA^{2}\right.$ ${ }^{112}$ ), basically within its uncertainty. However, no experimental data was found for IPPC membranes for which the APL value is larger by $1.6 \AA^{2}$ than that of DPPC membrane due to the branching lipid tail of isopalmitic-acid.

In the presence of dioxane, the average APL values increased by more than $3 \AA^{2}$ for both DPPC and IPPC membranes. To understand the local disturbances caused by 1,4-dioxane molecules, phosphorous atoms which are within $5 \AA$ of dioxane molecules are selected from the VT-based APL calculation (see red columns in Figure 4 and Figure 5), where the probability distributions of the total and local APL values are calculated and shown in Figure 5. The average of such local APL values found to be significantly larger than that of the conventional APL (more than $8 \%$ growth), namely $70.27 \AA^{2}$ for DPPC and $71.69 \AA^{2}$ for IPPC membrane which means that the lipid heads are pushed away due to the vicinity of dioxane. It is worth mentioning that the total APL distributions of clear systems are in the range of 30 and $110 \AA^{2}$ while in the case of polluted systems 30 and $120 \AA^{2}$ and have an upper tail (shown in black in Figure 5) which can be well described using lognormal distribution. However, assumption of normal distribution and arithmetic mean as expected value results within $2 \AA^{2}$ of the expected values of APL obtained from the proper lognormal fit (Figure 5) making a reasonable trade-off of APL widely used in the literature ${ }^{102,113,30}$. 
Further analyzing the probability distribution functions of Figure 5, the APL value with the highest probability (APL mode) is $57.48 \AA^{2}$ and $58.60 \AA^{2}$ for the clear DPPC and IPPC systems, respectively, while they shifted towards larger APL values in the case of polluted system $\left(62.31 \AA^{2}\right.$ for DPPC and $63.24 \AA^{2}$ for IPPC).

Volume per lipid, membrane thickness, isothermal compressibility modulus and deuterium order parameter

Volume per lipid (VPL), membrane thickness $\left(\mathrm{D}_{\mathrm{HH}}\right)$, isothermal compressibility $\left(\mathrm{K}_{\mathrm{A}}\right)$ and deuterium order parameter $\left(\mathrm{S}_{\mathrm{CD}}\right)$ have also been calculated based on the equations collected in Table 1. Calculated membrane parameters are shown in Figure 6 for the simulated membrane systems to further understand the effect of 1,4-dioxane pollution. To the best of our knowledge, experimental data is available only for DPPC composed membranes where the calculated results are in excellent agreement with literature values. For example, the relative deviation of VPL and $\mathrm{D}_{\mathrm{HH}}$ values are less than $2 \%$ and $3 \%$, respectively. $\left(\mathrm{VPL}_{\text {calc }}=1214 \pm 0,23 \AA^{3}, \mathrm{VPL}_{\mathrm{exp}}=1232 \AA^{3114}, \mathrm{D}_{\mathrm{HH} \text {,calc }}=37.2 \pm 4.5\right.$ $\left.\AA, \mathrm{D}_{\mathrm{HH}, \exp }=38.3 \AA^{114}\right)$.
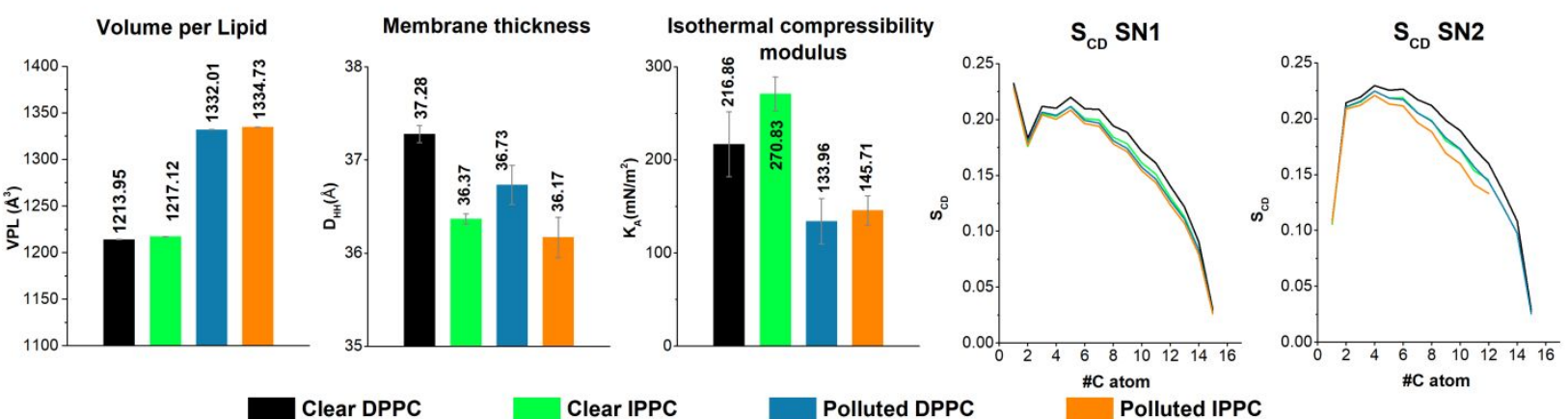

Figure 6 Volume per lipid (VPL in $\left.\AA^{3}\right)$, Membrane thickness $\left(\mathrm{D}_{\mathrm{HH}}\right.$ in $\AA$ ), Isothermal Compressibility $\left(\mathrm{K}_{\mathrm{A}}\right.$ in $\left.\mathrm{mN} / \mathrm{m}^{2}\right)$ and Deuterium Order Parameter $\left(\mathrm{S}_{\mathrm{CD}}\right)$ of the investigated PC membrane - 1,4-dioxane systems. Clear DPPC and IPPC membrane parameters are indicated with black and green respectively, while polluted DPPC-s are IPPC membranes are indicated with blue and orange, respectively.

Furthermore, a good agreement can be found also for calculated $\mathrm{S}_{\mathrm{CD}}$ values (for details see SFigure 3 in Supplementary Information), as their average relative deviations from experimental data measured in liquid-crystalline phase 115 are around $7 \%$. The largest relative deviation was obtained in the case of compressibility modulus $\left(\mathrm{K}_{\mathrm{A} \text {,calc }}=216.9 \pm 34.8 \mathrm{mN} / \mathrm{m}^{2}, \mathrm{~K}_{\mathrm{A} \text {,exp }}=231 \pm 20\right.$ $\mathrm{mN} / \mathrm{m}^{2}{ }^{114}$ ), which is close to $10 \%$, but uncertainties of $\mathrm{K}_{\mathrm{A}}$ in experiment and in calculation are still in overlap, therefore our computed results are in acceptable range.

As seen from Figure 6, the computed APL, VPL and compressibility modulus are significantly higher in the case of IPPC membranes than those for DPPC, while the membrane thickness of IPPC membrane is significantly lower, along with the orderliness of the lipid tails 
measured by $\mathrm{S}_{\mathrm{CD}}$. Since the branched lipids need larger space in the middle of the bilayer leading to larger disorder, resulting in the reduction of the relative length of such fatty acids. As the relative length of such lipids shortens, their spatial space requirement grows, increasing the APL and VPL of the IPPC composed membranes. The hereby obtained results also agree well with the previous simulations of IPPC and DPPC ${ }^{9}$ (IPPC was noted as $14-\mathrm{MpPPC}$ there).

The presence of the pollutant affects both membranes in a similar way, although the degree of the change is different. 1,4-dioxane pollution led to a significant rise in both APL and VPL while the thickness of the membrane, the compressibility modulus and the orderliness of the lipid chains decreased. As clear DPPC was found to be in liquid-crystalline phase and the $\mathrm{S}_{\mathrm{CD}}$ values of clear IPPC membranes is slightly lower than DPPC, we can assume that it can also be found in $\mathrm{L}_{\alpha}$ phase. As an effect of the pollution the lipid tails can be found in an even more disordered structure, thus we can be certain that the pollution does not induce phase transition. As mentioned before in the case of mass density profiles, 1,4-dioxane penetrates inside the membrane as deep as the ester groups (Region III) and the pollutant pushes away the membrane headgroups and lipid tails from each other leading to the swelling of the membrane which was already mentioned in the case of APL diagrams (Figure 4 and Figure 5). This swelling leads to a larger available space for the lipid tails, thus turning into a more disordered state with shorter relative length, affecting the membrane thickness. As IPPCs were found in an initially more disordered state, its change in the orderliness of the lipid chains and membrane thickness is less remarkable compared to the change for DPPC.

The most drastic change was found in the case of compressibility modulus. While the clear IPPC membrane has significantly higher $\mathrm{K}_{\mathrm{A}}$ compared to the clear DPPC membrane $\left(\mathrm{K}_{\mathrm{A}, \mathrm{DPPC} \text { clear }}=\right.$ $216.9 \pm 34.9 \mathrm{mN} / \mathrm{m}, \mathrm{K}_{\mathrm{A}, \mathrm{IPPC} \text { clear }}=270.8 \pm 18.14 \mathrm{mN} / \mathrm{m}$ ), these values dropped for both membranes to similar value $\left(\mathrm{K}_{\mathrm{A}, \mathrm{DPPC} \text { polluted }}=174.8 \pm 19.1 \mathrm{mN} / \mathrm{m}, \mathrm{K}_{\mathrm{A}, \mathrm{IPPC} \text { polluted }}=179.7 \pm 17.0 \mathrm{mN} / \mathrm{m}\right)$ due to the 1,4-dioxane pollution. This is an interesting finding in the light of the definition of $\mathrm{K}_{\mathrm{A}}$, since compressibility modulus is a quantity for the response of the membrane area to tension, which may come from environmental stress, like perturbations, or the addition of molecules to one of the membrane leaflets ${ }^{116}$. Therefore, in this case, smaller $\mathrm{K}_{\mathrm{A}}$ values mean smaller resistance towards environmental stress. This is in contradiction with the previous literature about branching fatty acids, that iso-branching would increase the tolerance of the cell membranes to toxic compounds ${ }^{10}$ and it shows no sign of enhancing the structural stability of the membrane-like phytanyl and phitanoyl ${ }^{11}$.

\section{Water orientational profiles and the determination of the interfacial regions}

Previous studies proved that based on the orientational order of the water molecules, four different regions can be separated in the membrane interface ${ }^{23,22,24}$. By means of orientational functions, charge densities and water density profiles, we have defined the bulk phase and these four 
different regions in the interfacial area, which are in line with the Marrink and Berendsen model ${ }^{27}$. Data will only be discussed regarding the negative side of the membrane normal $Z$. As the interface normal is fixed to point from the negative side to the positive, and $\alpha$ can be in the range of 0 and $180^{\circ}$, opposite orientations can be observed on the two sides of the membrane in the case of dipole orientation distribution. If the molecules do not have preferential alignment, then $\Phi_{\alpha}(Z), \Phi_{\beta}(Z)$ and $\Phi_{\gamma}(Z)$ are fluctuating around 0 . If $\Phi_{\beta}(Z)$ is lower than 0 , it indicates a more perpendicular, and if $\Phi_{\beta}(Z)$ larger than 0 , it indicates a more parallel alignment of the normal vector with the membrane surface. For $\Phi_{\gamma}(Z)$ the opposite is true, thus if $\Phi_{\gamma}(Z)$ is lower than 0 , it indicates that the H-H vector can be found in a more parallel alignment with the membrane surface, and if its larger than 0 it is in a more perpendicular alignment with the membrane surface.

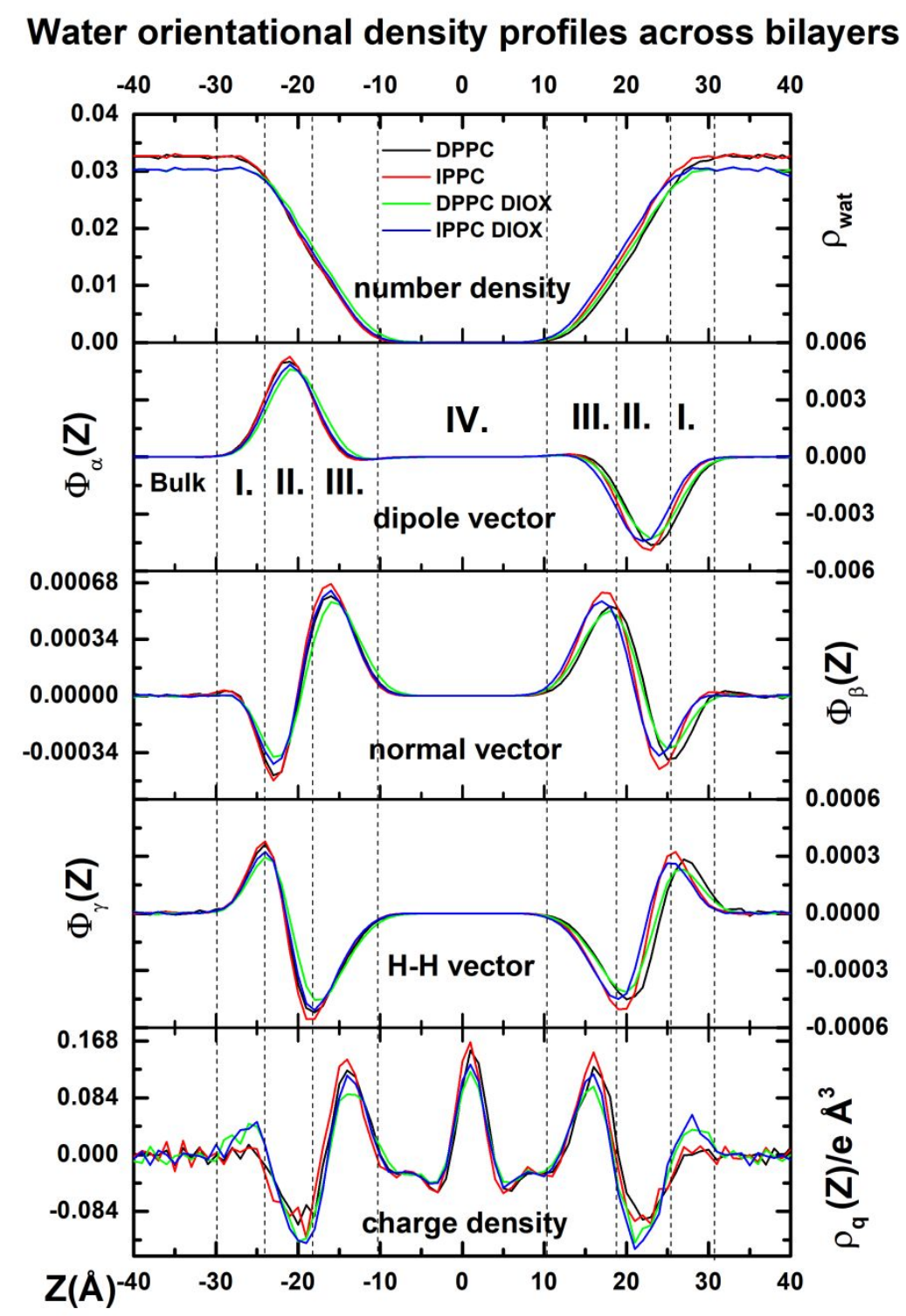

Figure 7 Profiles of number density and orientational functions of water and total charge density profile across the membrane/water interfaces. The $\mathrm{Z}=0$ corresponds to the center of the membrane. The dashed vertical lines indicate the borders between the regions of the interface. The results for clear DPPC and IPPC membranes are shown as black and red solid lines, respectively. The results for the DPPC and IPPC membrane in the presence of 1,4-dioxane are depicted by green and blue solid lines.

The number density, the orientational function of the dipole, normal and $\mathrm{H}-\mathrm{H}$ vector as well 
as the charge density profile of water molecules are shown in Figure 7. In the bulk phase, all these orientational functions are practically zero which in this case means there is no preferred direction for water molecules. Since the phosphatidylcholine headgroups contain negatively charged phosphate and positively charged tetramethylammonium groups, they determine mainly the orientation of the dipole moments of water molecules as they preferentially turn towards the negatively charged groups. Dipole, normal and $\mathrm{H}-\mathrm{H}$ vectors are all sensitive to the presence of charged headgroups, therefore their orientational functions can be used for determining the border of the bulk phase (Figure 7) thus defining the outer border of Region I. Since the orientational function of $\mathrm{H}-\mathrm{H}$ vector, $\Phi_{\gamma}(Z)$, is the most responsive to the structural changes, the borders of the Region II are defined by its stationary points, which coincidence with the inflexion points of the orientational function of the dipole vector $\Phi_{\alpha}(Z)$. Finally, the border between Region III and IV can be given by the coordinate where $\Phi_{\alpha}(Z)$ becomes zero again.

In the first interfacial region (noted as Region I), water density started to decrease slowly as seen in the top panel of Figure 7. $\Phi_{\beta}(Z)$ almost reached a minimum here, while $\Phi_{\alpha}(Z)$ showed quick growth, and $\Phi_{\gamma}(Z)$ reached a maximum, which was chosen as a border of this region. These changes manifested as the plane of water molecules are more likely to be found in a perpendicular orientation relative to the bilayer. The increasement of $\Phi_{\alpha}(Z)$ indicates that the dipole moments of water molecules start to point towards the phosphate groups. For the polluted systems, Region I is more positively charged while the clear system showed no charge accumulation here.

In the second region, $\Phi_{\alpha}(Z)$ reached a maximum, the orientational function of the normal vector linearly increased while $\Phi_{\gamma}(Z)$ decreased as getting closer to the middle of the membrane. This region is negatively charged for each system while this effect is more pronounced for the polluted systems resulting a more polarized outer layer for these membranes. Here, the dipole moment vectors enclose around $\sim 0^{\circ}$ with the surface normal, which means that the dipoles are pointing towards the membrane surface.

In the third region, water density vanished slowly and the decrease of $\Phi_{\alpha}(Z)$, as well as the increase of $\Phi_{\gamma}(Z)$ to zero, indicate that water molecules are slowly losing their orientational preference. Before the vanish of the orientational preference occurring at the inner part of Region III, the presence of the peak of $\Phi_{\beta}(Z)$ indicates that water molecules are taking a more planar alignment due to the presence of the ester groups.

In the central region (Region IV) no preferred orientations for water molecules can be seen based on the orientational profiles most probably due to the low statistical sampling of water molecules.

\section{1,4-dioxane orientational profiles along the membrane normal}


Similar to water, the orientational preference of 1,4-dioxane had been quantitatively described by orientational functions of the O-O $\left(\Phi_{\alpha}(Z)\right)$ and normal vector $\left(\Phi_{\beta}(Z)\right)$ as a function of the distance from the center of mass of the membrane (Figure 8). When $\Phi_{\beta}$ is lower than 0 , it indicates perpendicular, if larger, parallel alignment of the normal vector of the pollutant molecule with the membrane surface. DPPC and IPPC membranes have almost identical features for the number density profile of dioxane, as well as for the orientational profiles of the O-O and normal vectors. Orientational function of the O-O vector is centrosymmetric having minima at the border of Region I and II and at about $Z=-5 \AA$ (in Region IV) as well as maximum at $Z=-15 \AA$ (in Region III), while the orientational function of the normal vector is symmetric with a minimum at around $|Z|=8 \AA$.

A structural manifestation of these profiles is that random orientations are preferred in the bulk phase, then the molecular planes are in a planar position with the bilayer surface in the first two interfacial regions. The turning of the O-O vector throughout Region III and IV indicates, that while 1,4-dioxanes approach the membrane, the molecules turn around their $y$ axis, then turn back into a planar position. This turning can also be observed from the direct examination of the trajectories. In the inner side of the ester groups of the bilayer, 1,4-dioxanes can be found more likely in a parallel position with the membrane interface. Between the two types of membranes, no significant differences can be inspected.

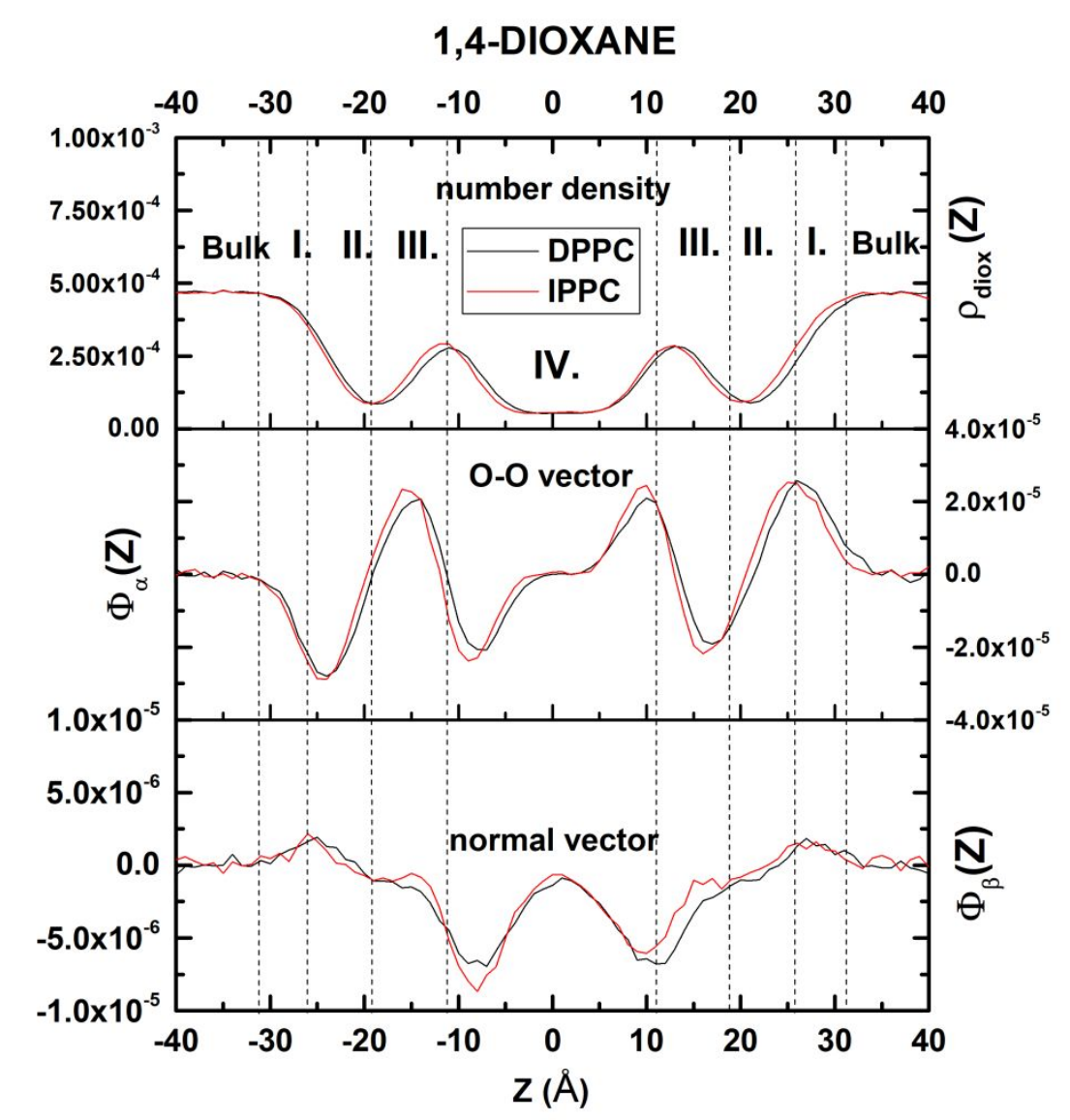

Figure 8 Orientational profiles of dioxane molecules across the membrane/water interfaces. The density of dioxane 
molecules is shown as a reference. The dashed vertical lines indicate the borders between the different regions.

\section{Orientational maps of water and 1,4-dioxane molecules in the membrane interfacial regions}

The two independent vectors ( $\alpha$ and $\delta$ ) of water and 1,4-dioxane molecules can be represented as maps which gives us a clearer view of their orientational preference in the different regions of the membrane/water interface. In the bulk phase and the first two regions of the water orientation maps significant differences cannot be observed between the two membranes (Figure 9), as expected from the presence of similar headgroups. In the bulk phase, no preferred orientation can be found. In the first region a slight orientational preference can be seen as the water molecules are more likely to be found in the $0 \leq \cos (\alpha) \leq 1$ region, while $\delta$ has no effect on the orientations, which means that the dipole moment vectors are starting to point towards the negatively charged headgroups of lipids, while the orientation of the plane of water molecules has no preference. This reference is more pronounced in the second region, but here the dipole orientations $(0.5 \leq \cos (\alpha) \leq 1)$ obviously show that the water molecules are in a perpendicular position with the membrane surface still without any $\delta$ preference. 
Bulk
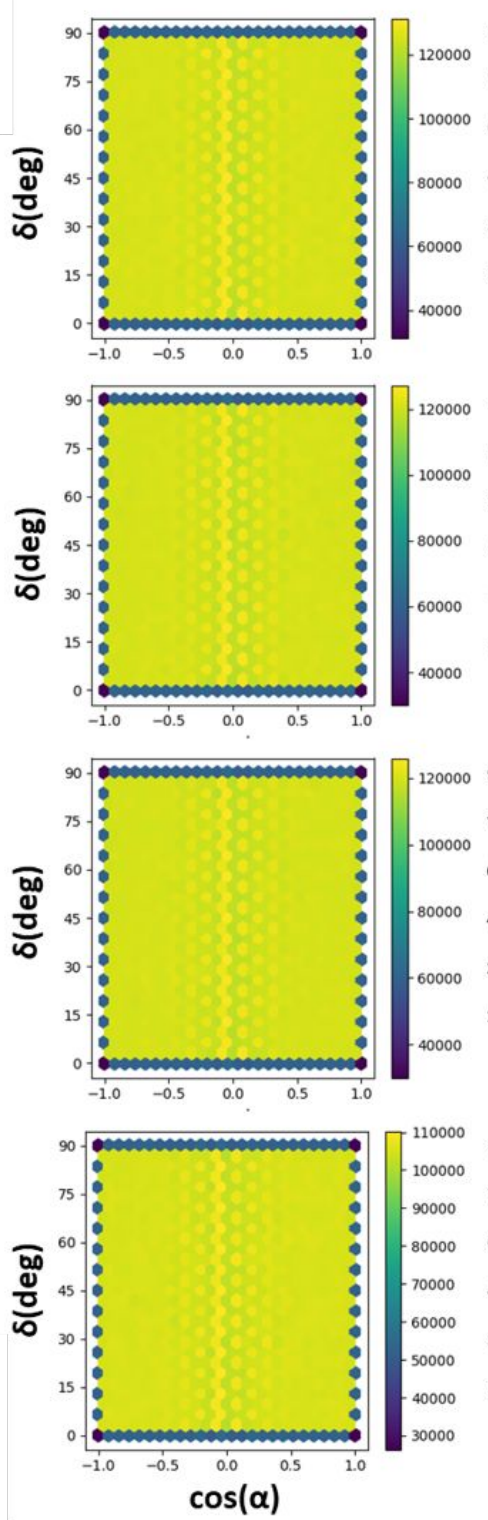

I.
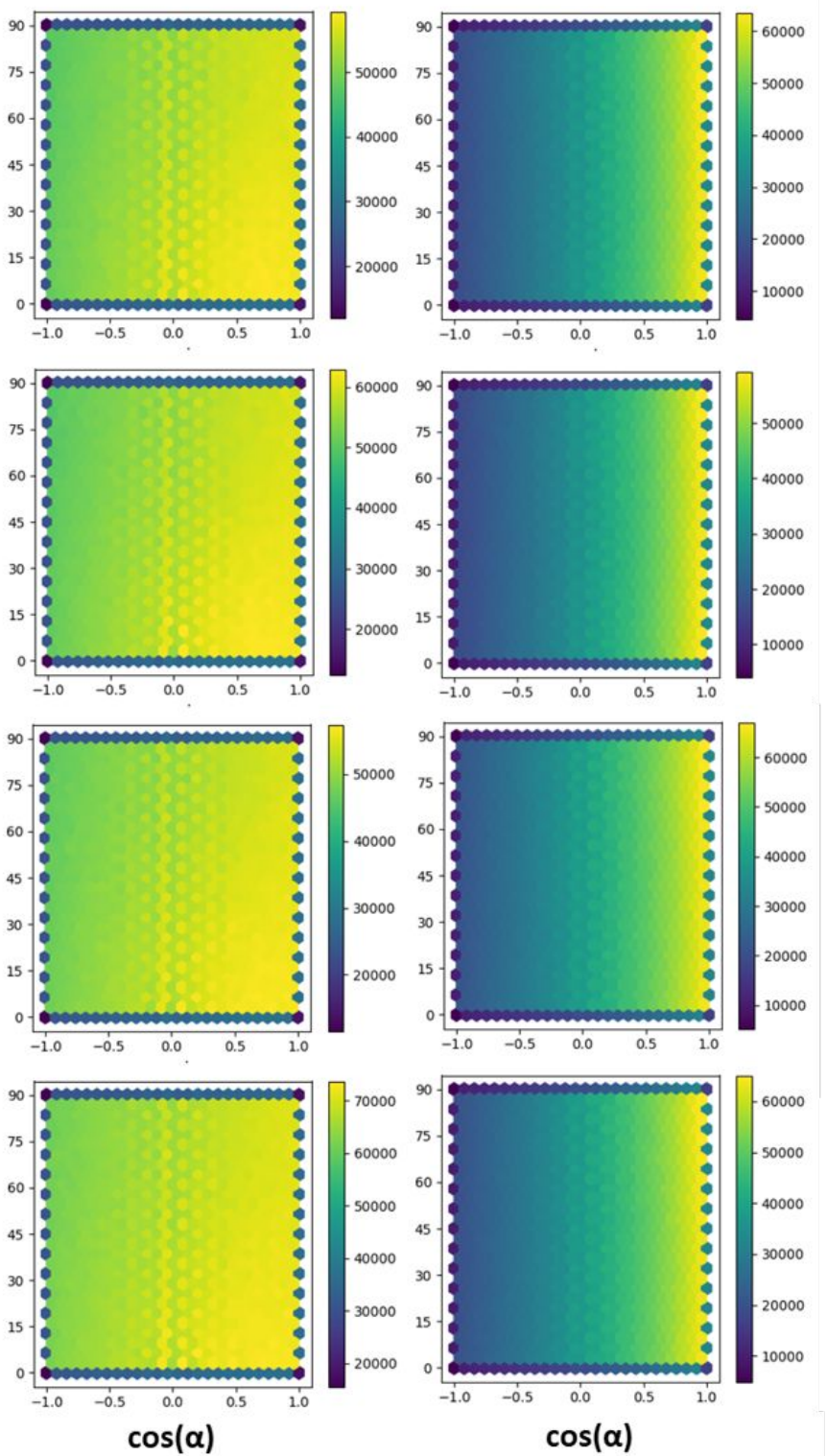

\section{ㅁํำ}

\section{กั}
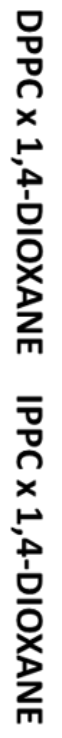

Figure 9 Orientational distribution maps of water molecules in the first three regions (Bulk and Region I and II) of the DPPC and IPPC membrane/water interfaces. While $\cos (\alpha)$ can be varied from -1 to $1, \delta$ is from $0^{\circ}$ to $90^{\circ}$ due to the symmetry of the water molecule. The color bars indicate the number of water molecules appearing in each region over $500 \mathrm{~ns}$ total simulation.

Angular distributions of water molecules found in the interior regions (Region III and IV) of DPPC and IPPC membranes are shown in Figure 10. In the third membrane region, the orientational peak is at the same place for both membrane types where the likeliness of perpendicular orientation of the water plane $\left(60^{\circ} \leq \delta \leq 90^{\circ}\right)$ is the highest, while the dipole moment vector is parallel with the bilayer surface $(-0.5 \leq \cos (\alpha) \leq 0.5)$. At the same time, more water molecules can be found in this region in the case of clear IPPC membranes compared to clear DPPC membranes.

In the central region (Region IV) a distinct peak can be observed in the orientation of water molecules at $60^{\circ} \leq \delta \leq 90^{\circ}$ and $0.0 \leq \cos (\alpha) \leq-1$, which indicates a quite similar orientation as in the 
fourth region, but the dipole vector turns around as the molecules approach the middle of the bilayer. At the same time the amount of water molecules is only third as much in this region in the case of branching fatty acids compared to the DPPC membranes in clean system. As an effect of 1,4-dioxane pollution, this difference disappears: almost the same amount of water molecules appear both in the third region and in the middle of the bilayer of both membranes.
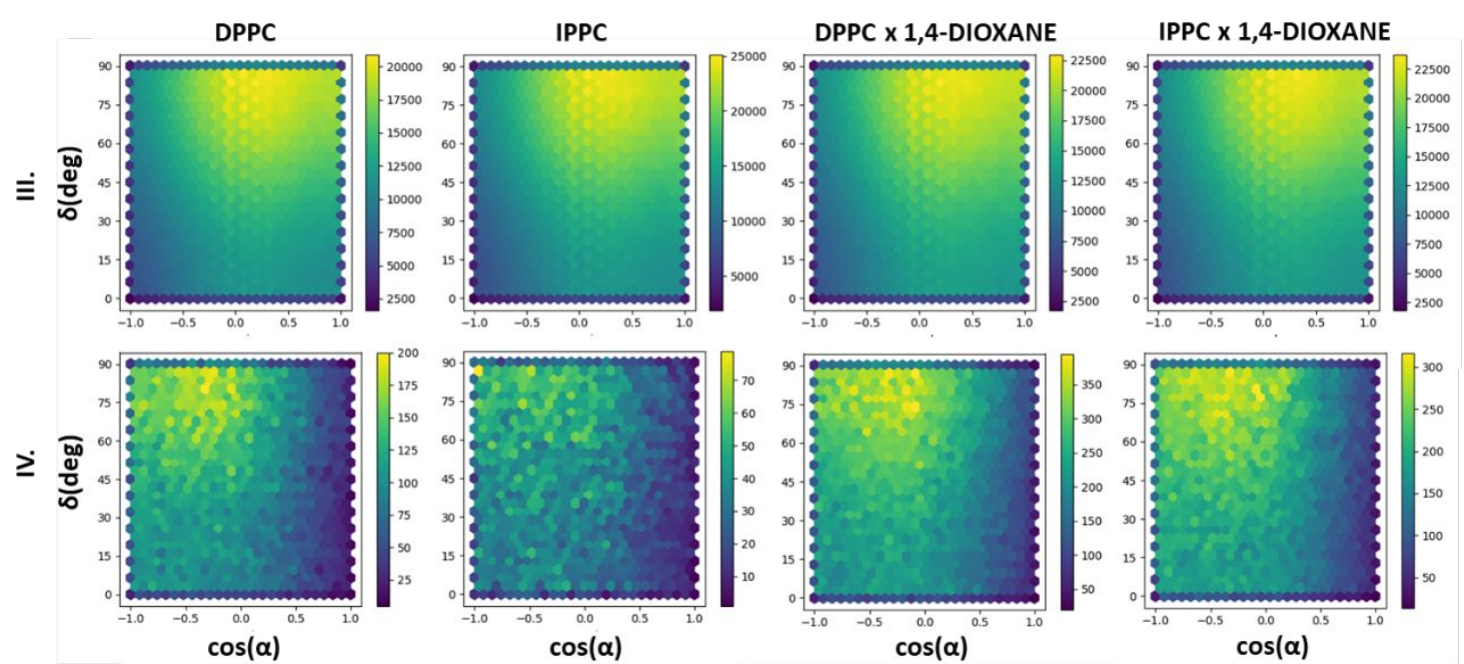

Figure 10 Orientational distribution maps of water molecules in the inner membrane regions (Region III and IV) of the DPPC and IPPC membrane/water interfaces. While $\cos (\alpha)$ can be varied from -1 to $1, \delta$ is from $0^{\circ}$ to $90^{\circ}$ due to the symmetry of the water molecules. The color bars indicate the number of water molecules appearing in each region over 500 ns total simulation. 
DPPC x 1,4-DIOXANE IPPC x 1,4-DIOXANE
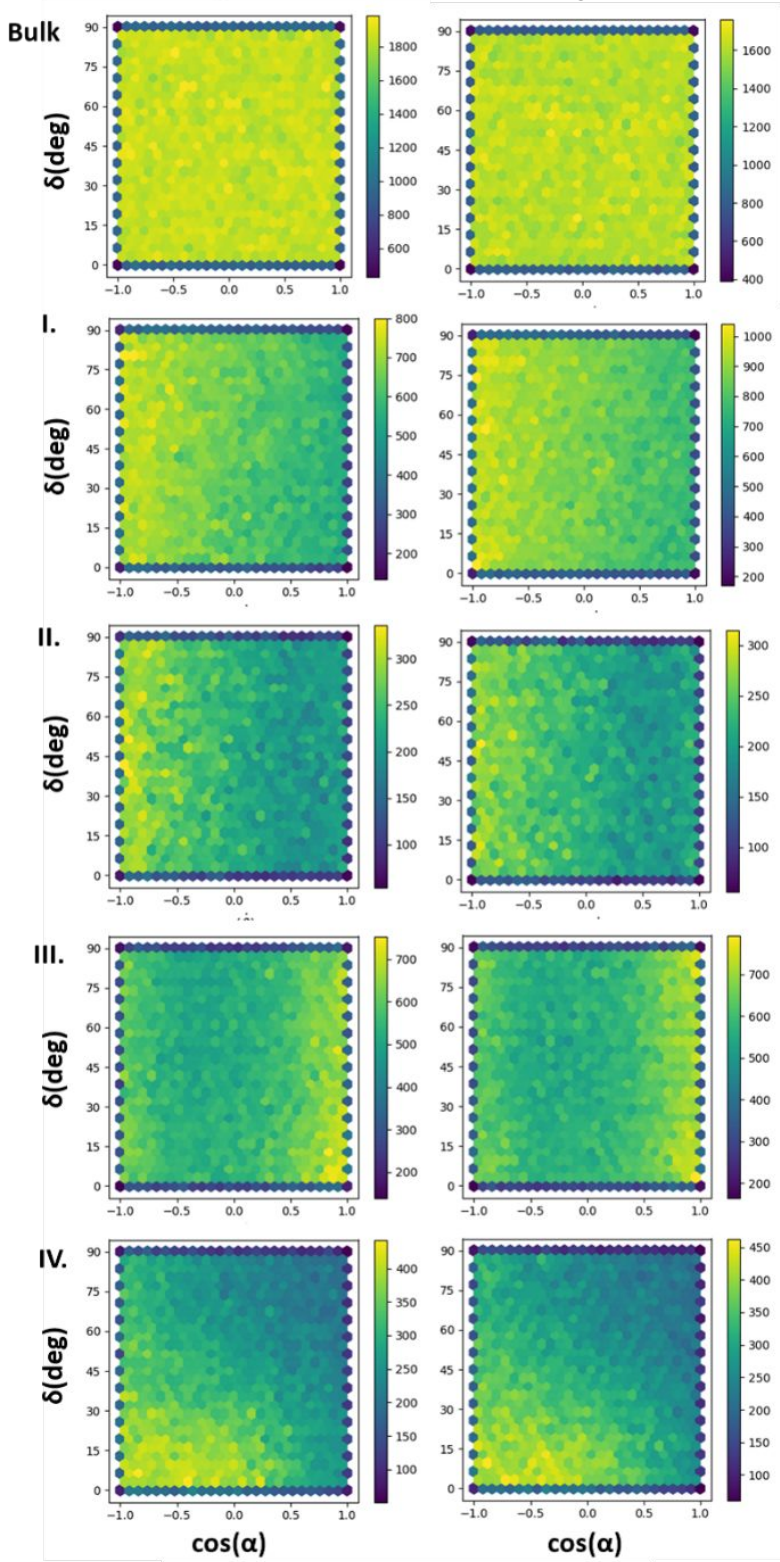

Figure 11 Orientational distribution maps of 1,4-dioxane molecules in the different region of the DPPC and IPPC membrane/water interfaces. While $\cos (\alpha)$ can be varied from -1 to $1, \delta$ is from $0^{\circ}$ to $90^{\circ}$ due to the symmetry of the dioxane molecule. The color bars indicate the number of dioxane molecules appearing in each region over $500 \mathrm{~ns}$ total simulation.

As Figure 11 shows, orientational maps of 1,4-dioxane molecules have very similar features for DPPC and IPPC membranes in each membrane/water region. As expected, dioxane molecules have random orientations in the bulk phase. In Region I and II, as it was also perceived from the orientational function profiles (see the middle panel of Figure 8), the O-O vectors of the pollutant molecules are parallel with the bilayer surface, but have no preference according to $\delta$. Similar is observed in Region III, but after the spin of the dioxane around its axis $y$.

In the central region (Region IV), the preferred orientation of dioxane molecules is within $0^{\circ}$ $\leq \delta \leq 30^{\circ}$ and $0 \leq \cos (\alpha) \leq-1.0$, which means that the $\mathrm{O}-\mathrm{O}$ vector is enclosing $90^{\circ}-45^{\circ}$ with the bilayer normal, while its normal vector is pointing towards the bilayer inner surface, which means a close to 
planar position of the pollutant molecule with the bilayer surface.

Free energy profile of the dioxane penetration through the membrane

Since DPPC and IPPC composed biomembrane models are changed similarly in the presence of 1,4-dioxane, metadynamics simulation of the dioxane penetration was carried out only in the case of DPPC composed membranes. The obtained free energy profiles along axis $Z$ are shown in Figure 12.

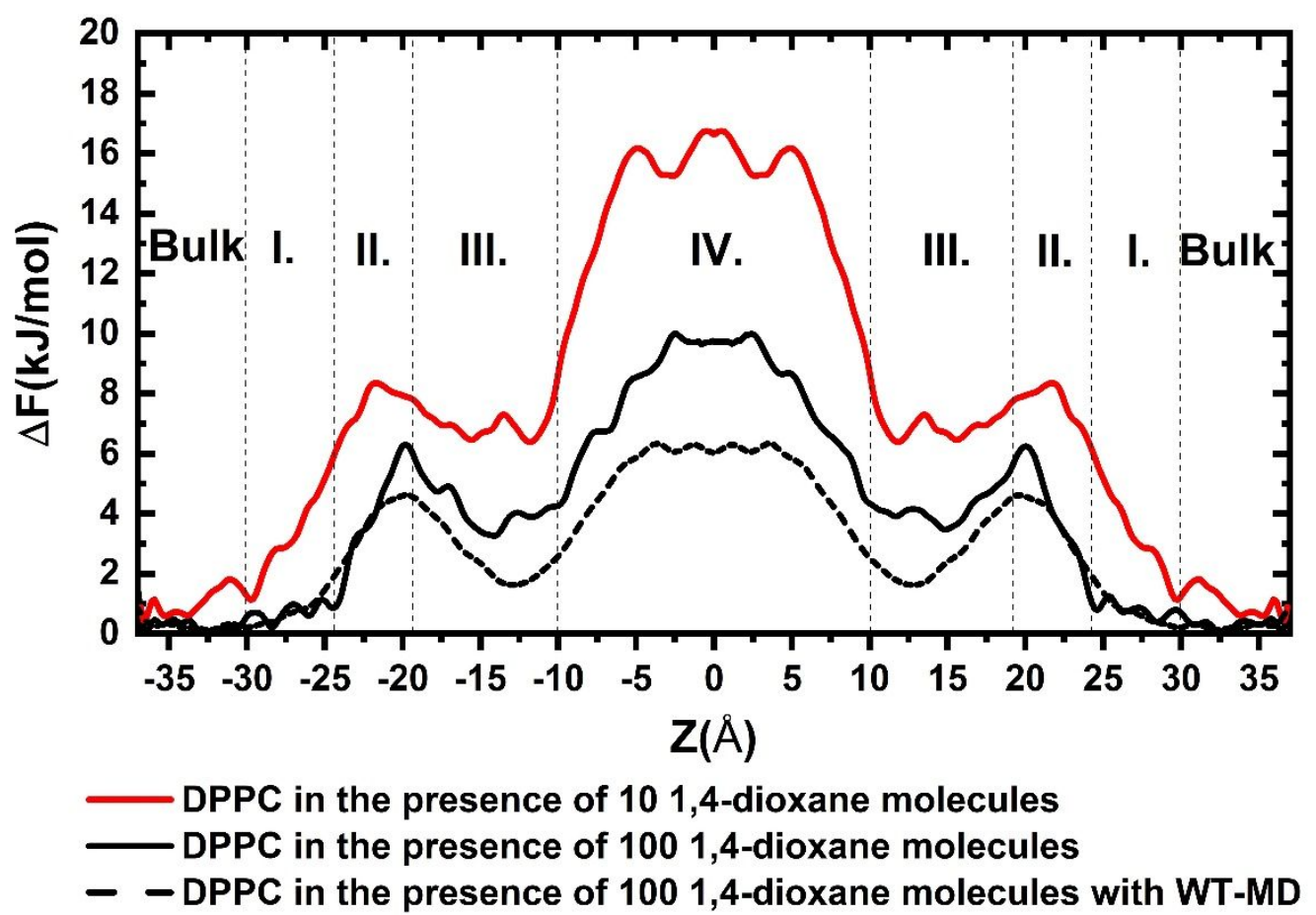

Figure 12 Free energy profile of 1,4-dioxane penetration into a DPPC bilayer. The black curve represents the results of the metadynamics simulations for the system having 100 1,4-dioxane molecules in the simulation (the average dioxane concentration is $75.102 \mathrm{~g} / \mathrm{dm}^{3}$ ), while red is for $7.510 \mathrm{~g} / \mathrm{dm}^{3}$ dioxane concentration). The dashed black curve indicates the results of the WT-MD simulation carried out for the system having 100 1,4-dioxane molecules. The estimated error of $\Delta \mathrm{F}$ is $4 \mathrm{~kJ} / \mathrm{mol}$.

The lowest free energy corresponds to the bulk water region $(|Z|>30 \AA)$ which is therefore the preferred state for 1,4-dioxane molecules and it has been chosen as the reference line. As 1,4dioxane approaches the bilayer surface $\Delta F$ starts to grow sharply until it reaches the head groups in Region II where the free energy profile reaches a local maximum. The starting point of the growth of free energy appears to be closer to the bilayer center for the more concentrated system by $5 \AA$ while the relative free energy values appear to be somewhat higher for the diluted system $\left(\Delta F_{\text {diluted }}\right)$ than that of the concentrated one $\left(\Delta F_{\text {conc }}\right)$. This means that the penetrating dioxane molecules make the membrane more accessible for additional pollutant molecules. The height and the position of the local free energy barrier at the sight of the head groups shows only a weak concentration dependence: $\Delta F_{\text {diluted }}=8.3 \mathrm{~kJ} / \mathrm{mol}$ at $|Z|=21.8 \AA$ and $\Delta F_{\text {conc }}=6.5 \mathrm{~kJ} / \mathrm{mol}$ at $|Z|=19.7 \AA$ for the concentrated system. As dioxane penetrates inside the membrane (Region III) the free energy profile reaches a metastable 
state where $\Delta F_{\text {conc }}=6.4 \mathrm{~kJ} / \mathrm{mol}$ and $\Delta F_{\text {diluted }}=3.3 \mathrm{~kJ} / \mathrm{mol}$, which corresponds to the site of the ester groups $(|Z|=16-11 \AA)$. The local maximum in dioxane density - which was experienced previously in the case of mass density profiles (bottom of Figure 3) - is consistent with this free energy basin. As 1,4-dioxane approaches the bilayer center (Region IV) free energy starts to grow steeply again until it reaches the global free energy maximum in the bilayer center where $\Delta F_{\text {conc }}=16.7 \mathrm{~kJ} / \mathrm{mol}$ and $\Delta F_{\text {diluted }}=10.4 \mathrm{~kJ} / \mathrm{mol}$.

To test the results of the metadynamics simulations an additional WT-MD simulation have been carried out for the higher dioxane concentration as seen in Figure 12 (shown as dashed line). Since WT-MD profile agrees with that of the previous metadynamics simulation (shown as solid black line in Figure 12) within the chemical accuracy, the barrier height of the penetration at the bilayer center appears at $6 \mathrm{~kJ} / \mathrm{mol}$.

Molecules with both hydrophilic and hydrophobic components demonstrate a sinusoidal pattern where the free energy decreases near the interface (e.g. Regions II and III) and increases at the center of the membrane bilayer. This pattern is commonly witnessed among biomolecules such as methanol ${ }^{117,118}$ or acetone ${ }^{119}$, which is similar to the obtained results for 1,4-dioxane.

Due to the relatively small height of the free energy barrier, DPPC membranes can be permeable for 1,4-dioxane which effect can be further enhanced by increasing dioxane concentration. This allows us to speculate that the presence of dioxane might also make the membrane more permeable for additional molecules, similar to the effects of alcohol ${ }^{120}$.

\section{Conclusion}

The effect of 1,4-dioxane on biomembrane models composed of DPPC - and its branched isomer - IPPC was thoroughly analyzed by the means of MD simulations. We have been focusing on structural changes induced by the pollutant - inside the membrane and also in its vicinity by creating orientational maps of water and 1,4-dioxane molecules - as well as energetics of its penetration.

We have thoroughly analyzed the effects of 1,4-dioxane on the membrane structural parameters, such as mass density distribution, $\mathrm{APL}, \mathrm{VPL}, \mathrm{S}_{\mathrm{CD}}, \mathrm{D}_{\mathrm{HH}}, \mathrm{K}_{\mathrm{A}}$. In pollutant free environment, as an effect of the branching found in one of the IPPC acyl chains, IPPC composed membranes have significantly higher APL, VPL and $\mathrm{K}_{\mathrm{A}}$ values compared to those of DPPC, while its $\mathrm{D}_{\mathrm{HH}}$ and $\mathrm{S}_{\mathrm{CD}}$ values are significantly lower. To the presence of 1,4-dioxane both membranes respond the same way. As observable from the mass density profiles, 1,4-dioxane can penetrate inside the membranes, where it appears at more than half of its bulk phase density at the inner site of the ester groups of the fatty acid chains. As an effect the pollutant pushes away the phospholipids from each other, leading to the swelling of the membrane. This phenomena can also be observed from the growth of local APL 
in the vicinity of 1,4-dioxane. This created disturbance in the distribution of phospholipids leads to an overall lateral growth of the biomembranes, a more disordered state of the lipid tails and thinning of the bilayer. This overall change reduces the stress resistance of the biomembrane $\left(\mathrm{K}_{\mathrm{A}}\right)$, which may initiate a serious cell-damaging process induced by 1,4-dioxane pollution by increasing its permeability.

Four regions of the membrane/water interface were distinguished by the orientational functions based on the orientational preference of the local vectors (dipole, $\mathrm{H}-\mathrm{H}$ and normal vector) bound to the water molecules, which are equivalent to the membrane regions proposed by Marrink and Berendsen. In the interfacial regions (Region I, II, III and IV) starting from about $30 \AA$ from the center of the bilayer, not only water but also dioxane molecules can be found in highly ordered states which have been analyzed via orientational maps. From these maps it can also be understood clearly that dioxane is hydrated throughout its dissolution, as water density also increased in the membrane interior. The free energy of 1,4-dioxane penetration into DPPC membranes at two different concentrations (75.102 $\mathrm{g} / \mathrm{dm}^{3}$ and $7.5102 \mathrm{~g} / \mathrm{dm}^{3}$ ) was obtained using metadynamics parallel with well-tempered metadynamics to understand the method dependence of the profile. The results of the two methods are found to be consistent with each other.

By comparing the results of the orientational maps and the free energy calculations the penetration mechanism of 1,4-dioxane into DPPC composed biomembranes can be described in detail. The pollutant molecule can be found with the lowest free energy in the bulk phase which is parallel with the mass density profiles obtained from classical molecular dynamics simulations, where the highest concentration of dioxane was found to be at this region. In Region I the plane of dioxane is preferred to be parallel to the membrane surface, and at the same time a growth in free energy is observed. In Region II the pollutant molecule twists around its normal vector, the free energy of the mechanism reaches a peak, and dioxane density drops down. In Region III 1,4-dioxane is found to be parallel with the bilayer surface. Meanwhile, its density increases, and the free energy gets lower, indicating, that this is a metastate for the penetration mechanism. Here, dioxane can establish weak induced dipole-dipole interactions with the ester groups, pushing away the phospholipid molecules from each other. As the pollutant reaches Region IV, it turns around its normal vector, so that the $\mathrm{O}-\mathrm{O}$ vector is pointing towards the bilayer surface. In the center free energy reaches a global maximum, while density of 1,4-dioxane reaches a global minimum. The obtained free energy profiles of the penetration shows concentration dependence. The relative free energy value is found to be distinctly higher for the diluted system than that of the concentrated one which means that 1,4-dioxane makes the membrane more accessible for additional pollutant molecules.

\section{Acknowledgments}


This research was supported by the European Union and the Hungarian State, co-financed by the European Regional Development Fund in the framework of the GINOP-2.3.4-15-2016-00004 project, aimed to promote the cooperation between the higher education and the industry. Milán Szőri gratefully acknowledges the financial support by the János Bolyai Research Scholarship of the Hungarian Academy of Sciences (BO/00113/15/7) and the additional financial support (Bolyai+) from the New National Excellence Program of the Ministry of Human Capacities (ÚNKP-18-4ME/4). The GITDA (Governmental Information-Technology Development Agency, Hungary) and Péter Huszák are also gratefully acknowledged for allocating and managing computing resources used in this work.

\section{Supporting Information Available}

Included is a diagram of the comparision of calculate and experimental electron density profile of DPPC membranes, the radial distribution function of the penetrated 1,4-dioxane molecules and the comparison of the calculated and experimental $\mathrm{S}_{\mathrm{CD}}$ profiles of DPPC membranes. 


\section{References}

(1) Zhang, Y.; Lukacova, V.; Bartus, V.; Balaz, S. Structural Determinants of Binding of Aromates to Extracellular Matrix: A Multi-Species Multi-Mode CoMFA Study. Chem. Res. Toxicol 2007, 20, 11-19.

(2) Bemporad, D.; Essex, J. W.; Luttmann, C. Permeation of Small Molecules Through a Lipid Bilayer: A Computer Simulation Study. J. Phys. Chem. B 2004, 108, 4875-4884.

(3) Palancyová, M.; Berka, K.; Otyepka, M. Convergence of Free Energy Profile of Coumarin in Lipid Bilayer. J. Chem. Theory Comput. 2012, 8, 1200-1211.

(4) Mayne, C. G.; Arcario, M. J.; Mahinthichaichan, P.; Baylon, J. L.; Vermaas, J. V.; Navidpour, L.; Wen, P. C.; Thangapandian, S.; Tajkhorshid, E. The Cellular Membrane as a Mediator for Small Molecule Interaction with Membrane Proteins. Biochim. Biophys. Acta Biomembr. 2016, 1858, 2290-2304.

(5) Wu, N.; Palczewski, K.; Müller, D. J. Vertebrate Membrane Proteins : Structure , Function , and Insights from Biophysical Approaches. Pharmacol. Rev. 2008, 60, 43-78.

(6) Parsons, J. B.; Rock, C. O. Progress in Lipid Research Bacterial Lipids : Metabolism and Membrane Homeostasis. Prog. Lipid Res. 2013, 52, 249-276.

(7) Kaneda, T. Iso- and Anteiso-Fatty Acids in Bacteria: Biosynthesis, Function, and Taxonomic Significance. Microbiol. Rev. 1991, 55, 288-302.

(8) Poger, D.; Caron, B.; Mark, A. E. Effect of Methyl-Branched Fatty Acids on the Structure of Lipid Bilayers. J. Phys. Chem. B 2014, 118, 13838-13848.

(9) Lim, J. B.; Klauda, J. B. Lipid Chain Branching at the Iso- and Anteiso-Positions in Complex Chlamydia Membranes : A Molecular Dynamics Study. Biochim. Biophys. Acta-Biomembr. 2011, 1808, 323-331.

(10) Frostegard, A.; Tunlid, A.; Baath, E. Phospholipid Fatty Acid Composition, Biomass, and Activity of Microbial Communities from Two Soil Types Experimentally Exposed to Different Heavy Metals. Appl. Environ. Microbiol. 1993, 59, 3605-3617.

(11) Shinoda, W.; Mikami, M.; Baba, T.; Hato, M. Molecular Dynamics Study on the Effect of Chain Branching on the Physical Properties of Lipid Bilayers: Structural Stability. J. Phys. Chem. B 2003, 107, 14030-14035.

(12) Shinoda, W.; Mikami, M.; Baba, T.; Hato, M. Molecular Dynamics Study on the Effects of Chain Branching on the Physical Properties of Lipid Bilayers: 2. Permeability. J. Phys. Chem. B 2004, 108, 9346-9356.

(13) Martelli, F.; Ko, H.-Y.; Borallo, C. C.; Franzese, G. Structural Properties of Water Confined by Phospholipid Membranes. Front. Phys. 2018, 13, 136801.

(14) Gawrisch, K.; Ruston, D.; Zimmerberg, J.; Parsegian, V. A.; Rand, R. P.; Fuller, N. 
Membrane Dipole Potentials, Hydration Forces, and the Ordering of Water at Membrane Surfaces. Biophys. J. 1992, 61, 1213-1223.

(15) Cheng, J.-X.; Pautot, S.; Weitz, D. A.; Xie, X. S. Ordering of Water Molecules between Phospholipid Bilayers Visualized by Coherent Anti-Stokes Raman Scattering Microscopy. Proc. Natl. Acad. Sci. U. S. A. 2003, 100, 9826-9830.

(16) Fukuma, T.; Higgins, M. J.; Jarvis, S. P. Direct Imaging of Individual Intrinsic Hydration Layers on Lipid Bilayers at Ångstrom Resolution. Biophys. J. 2007, 92, 3603-3609.

(17) Yeghiazaryan, G. A.; Poghosyan, A. H.; Shahinyan, A. A. The Water Molecules Orientation around the Dipalmitoylphosphatidylcholine Head Group: A Molecular Dynamics Study. Physica A 2006, 362, 197-203.

(18) Inoue, K.; Singh, P. C.; Nihonyanagi, S.; Yamaguchi, S.; Tahara, T. Cooperative HydrogenBond Dynamics at a Zwitterionic Lipid/Water Interface Revealed by 2D HD-VSFG Spectroscopy. J. Phys. Chem. Lett. 2017, 8, 5160-5165.

(19) Kasson, P. M.; Lindahl, E.; Pande, V. S. Water Ordering at Membrane Interfaces Controls Fusion Dynamics. J. Am. Chem. Soc. 2011, 133, 3812-3815.

(20) Perera, L.; Essmann, U.; Berkowitz, M. L. Role of Water in the Hydration Force Acting between Lipid Bilayers. Langmuir 1996, 12, 2625-2629.

(21) Higgins, M. J.; Polcik, M.; Fukuma, T.; Sader, J. E.; Nakayama, Y.; Jarvis, S. P. Structured Water Layers Adjacent to Biological Membranes. Biophys. J. 2006, 91, 2532-2542.

(22) Jedlovszky, P.; Vincze, A.; Horvai, G. New Insight into the Orientational Order of Water Molecules at the Water/1,2-Dichloroethane Interface: A Monte Carlo Simulation Study. $J$. Chem. Phys. 2002, 117, 2271-2280.

(23) Jedlovszky, P.; Mezei, M. Orientational Order of the Water Molecules Across a Fully Hydrated DMPC Bilayer: A Monte Carlo Simulation Study. J. Phys. Chem. B 2001, 105, 3614-3623.

(24) Szőri, M.; Roeselová, M.; Jedlovszky, P. Surface Hydrophilicity-Dependent Water Adsorption on Mixed Self-Assembled Monolayers of C7-CH3 and C7-COOH Residues. A Grand Canonical Monte Carlo Simulation Study. J. Phys. Chem. C 2011, 115, 19165-19177.

(25) Neale, C.; Bennett, W. F. D.; Tieleman, D. P. Statistical Convergence of Equilibrium Properties in Simulations of Molecular Solutes Embedded in Lipid Bilayers. J. Chem. Theory Comput. 2011, 7, 4175-4188.

(26) Orsi, M.; Essex, J. W. Permeability of Drugs and Hormones through a Lipid Bilayer: Insights from Dual-Resolution Molecular Dynamics. Soft Matter 2010, 6, 3797-3808.

(27) Marrink, S. J.; Berendsen, H. J. C. Permeation Process of Small Molecules across Lipid Membranes Studied by Molecular Dynamics Simulations. J. Phys. Chem. 1996, 100, 16729- 
16738.

(28) Bemporad, D.; Luttmann, C.; Essex, J. W. Behaviour of Small Solutes and Large Drugs in a Lipid Bilayer from Computer Simulations. Biochim. Biophys. Acta 2005, 1718, 1-21.

(29) Parrill, A. L.; Lipkowitz, K. B. Reviews in Computational Chemistry 28; John Wiley \& Sons: New Jersey, 2015.

(30) Moradi, S.; Shahlaei, M. Shedding Light on the Structural Properties of Lipid Bilayers Using Molecular Dynamics Simulation: A Review Study. RSC Adv. 2019, 9, 4644-4658.

(31) Larsson, P.; Alskär, L. C.; Bergström, C. A. S. Molecular Structuring and Phase Transition of Lipid-Based Formulations upon Water Dispersion: A Coarse-Grained Molecular Dynamics Simulation Approach. Mol. Pharm. 2017, 14, 4145-4153.

(32) Gupta, R.; Rai, B. Molecular Dynamics Simulation Study of Skin Lipids: Effects of the Molar Ratio of Individual Components over a Wide Temperature Range. J. Phys. Chem. B 2015, 119, 11643-11655.

(33) Schindler, T.; Kröner, D.; Steinhauser, M. O. On the Dynamics of Molecular Self-Assembly and the Structural Analysis of Bilayer Membranes Using Coarse-Grained Molecular Dynamics Simulations. Biochim. Biophys. Acta - Biomembr. 2016, 1858, 1955-1963.

(34) Skjevik, Å. A.; Madej, B. D.; Dickson, C. J.; Lin, C.; Teigen, K.; Walker, R. C.; Gould, I. R. Simulation of Lipid Bilayer Self-Assembly Using All-Atom Lipid Force Fields. Phys. Chem. Chem. Phys. 2016, 18, 10573-10584.

(35) Friedman, R. Membrane-Ion Interactions. J. Membr. Biol. 2018, 251, 453-460.

(36) Nowroozi, A.; Shahlaei, M. A Coupling of Homology Modeling with Multiple Molecular Dynamics Simulation for Identifying Representative Conformation of GPCR Structures: A Case Study on Human Bombesin Receptor Subtype-3. J. Biomol. Struct. Dyn. 2017, 35, 250272.

(37) Pothula, K. R.; Solano, C. J. F.; Kleinekathöfer, U. Simulations of Outer Membrane Channels and Their Permeability. Biochim. Biophys. Acta - Biomembr. 2016, 1858, 17601771.

(38) Pokhrel, N.; Maibaum, L. Free Energy Calculations of Membrane Permeation: Challenges Due to Strong Headgroup-Solute Interactions. J. Chem. Theory Comput. 2018, 14, $1762-1771$.

(39) Hantal, G.; Fábián, B.; Sega, M.; Jójárt, B.; Jedlovszky, P. Effect of General Anesthetics on the Properties of Lipid Membranes of Various Compositions. Biochim. Biophys. Acta Biomembr. 2019, 1861, 594-609.

(40) Lundborg, M.; Wennberg, C. L.; Narangifard, A.; Lindahl, E.; Norlén, L. Predicting Drug Permeability through Skin Using Molecular Dynamics Simulation. J. Control. Release 2018, 
$283,269-279$.

(41) Lee, C. T.; Comer, J.; Herndon, C.; Leung, N.; Pavlova, A.; Swift, R. V.; Tung, C.; Rowley, C. N.; Amaro, R. E.; Chipot, C.; et al. Simulation-Based Approaches for Determining Membrane Permeability of Small Compounds. J. Chem. Inf. Model. 2016, 56, 721-733.

(42) Fabian, B.; Darvas, M.; Picaud, S.; Sega, M.; Jedlovszky, P. The Effect of Anaesthetics on the Properties of a Lipid Membrane in the Biologically Relevant Phase: A Computer Simulation Study. Phys. Chem. Chem. Phys. 2015, 17, 14750-14760.

(43) Jedlovszky, P.; Mezei, M. Calculation of the Free Energy Profile of H2O, O2, CO, CO2, NO, and $\mathrm{CHCl} 3$ in a Lipid Bilayer with a Cavity Insertion Variant of the Widom Method. J. Am. Chem. Soc. 2000, 122, 5125-5131.

(44) Klimovich, P. V; Shirts, M. R.; Mobley, D. L. Guidelines for the Analysis of Free Energy Calculations. J. Comput. Aided Mol. Des. 2015, 29, 397-411.

(45) Hansen, N.; Gunsteren, W. F. Van. Practical Aspects of Free-Energy Calculations: A Review. J. Chem. Theory Comput. 2014, 10, 2632-2647.

(46) Meral, D.; Provasi, D.; Filizola, M. An Efficient Strategy to Estimate Thermodynamics and Kinetics of G Protein-Coupled Receptor Activation Using Metadynamics and Maximum Caliber. J. Chem. Phys. 2018, 149, 224101.

(47) Torrie, G.; Valleau, J. Nonphysical Sampling Distributions in Monte Carlo Free-Energy Estimation: Umbrella Sampling. J. Comput. Phys. 1977, 23, 187-199.

(48) Dickson, B. M.; Lelie, T.; Stoltz, G.; Fleurat-lessard, P.; Supe, N. Free Energy Calculations : An Efficient Adaptive Biasing Potential Method. J. Phys. Chem. B 2010, 114, 5823-5830.

(49) Kirkwood, J. G. Statistical Mechanics of Fluid Mixtures. J. Chem. Phys. 1935, 3, 300-313.

(50) Vymetal, J.; Vondrásek, J. Metadynamics As a Tool for Mapping the Conformational and Free-Energy Space of Peptides - The Alanine Dipeptide Case Study. J. Phys. Chem. B 2010, $114,5632-5642$.

(51) Laio, A.; Parrinello, M. Escaping Free-Energy Minima. PNAS 2002, 99, 12562-12566.

(52) Barducci, A.; Bussi, G.; Parrinello, M. Well-Tempered Metadynamics: A Smoothly Converging and Tunable Free-Energy Method. Phys. Rev. Lett. 2008, 100, 020603.

(53) Zenker, M. J.; Borden, R. C.; Barlaz, M. A. Occurrence and Treatment of 1,4-Dioxane in Aqueous Environments. Environ. Eng. Sci. 2003, 20, 423-432.

(54) Agency for Toxic Substances \& Disease Registry. Toxicological profile for 1,4-dioxane https://www.atsdr.cdc.gov/toxprofiles/tp.asp?id=955\&tid=199 (accessed Apr 4, 2019).

(55) Karges, U.; Becker, J.; Püttmann, W. 1,4-Dioxane Pollution at Contaminated Groundwater Sites in Western Germany and Its Distribution within a TCE Plume. Sci. Total Environ. 2018, 619-620, 712-720. 
(56) Richardson, S. D.; Ternes, T. A. Water Analysis : Emerging Contaminants and Current Issues. Anal. Chem. 2011, 83, 4614-4648.

(57) Institute for Health and Consumer Protection. European Union Risk Assessment Report: 1,4Dioxane; European Communities, 2002.

(58) Occupational Toxicants: Critical Data Evaluation for MAK Values and Classification of Carcinogens; Greim, H., Ed.; Wiley-VCH Verlag: Weinheim, 2003; Vol. 20.

(59) Young, J. D.; Braun, W. H.; Gehring, P. J. Dose-Dependent Fate of 1,4-Dioxane in Rats. J. Toxicol. Environ. Health 1978, 4, 709-726.

(60) Göen, T.; Helden, F. Von; Eckert, E.; Knecht, U.; Drexler, H.; Walter, D. Metabolism and Toxicokinetics of 1,4 - Dioxane in Humans after Inhalational Exposure at Rest and under Physical Stress. Arch. Toxicol. 2015, 90, 1315-1324.

(61) Bennett, G. The Merck Index: An Encyclopedia of Chemicals, Drugs and Biologicals, 11th ed.; Budavari, S., Ed.; Merck: Rahway, N.J., USA, 2002; Vol. 30.

(62) Keith, L. H.; Walters, D. B. Compendium of Safety Data Sheets for Research and Industrial Chemicals, Part VII, Flavour and Fragance Substances; Zebovitz, T. C., Ed.; VCH Pub, 2002; Vol. 237.

(63) US Environmental Protection Agency. Treatment Technologies for 1,4-Dioxane: Fundamentals and Field Applications; Cincinnati, 2006.

(64) Street, J. J. Handbook of Environmental Fate and Exposure Data for Organic Chemicals, Volume 1-Large Production and Priority Pollutants; Howard, P. H., Sage, G. W., Jarvis, W. F., Gray, D. A., Eds.; CRC Press, 2010; Vol. 19.

(65) Frigini, E.; López Cascales, J.; Porasso, R. Molecular Dynamics Simulations of Glyphosate in a DPPC Lipid Bilayer. Chem Phis Lipids 2018, 213, 111-117.

(66) Wu, E. L.; Cheng, X.; Jo, S.; Rui, H.; Song, K. C.; Dávila-Contreras, E. M.; Qi, Y.; Lee, J.; Monje-Galvan, V.; Venable, R. M.; et al. CHARMM-GUI Membrane Builder - Toward Realistic Biological Membrane Simulations. J. Comput. Chem. 2014, 35, 1997-2004.

(67) Lee, J.; Cheng, X.; Swails, J. M.; Yeom, M. S.; Eastman, P. K.; Lemkul, J. A.; Wei, S.; Buckner, J.; Jeong, J. C.; Qi, Y.; et al. CHARMM-GUI Input Generator for NAMD, GROMACS, AMBER, OpenMM, and CHARMM/OpenMM Simulations Using the CHARMM36 Additive Force Field. J. Chem. Theory Comput. 2016, 12, 405-413.

(68) Khondker, A.; Dhaliwal, A.; Alsop, R. J.; Tang, J.; Backholm, M.; Shi, A. C.; Rheinstädter, M. C. Partitioning of Caffeine in Lipid Bilayers Reduces Membrane Fluidity and Increases Membrane Thickness. Phys. Chem. Chem. Phys. 2017, 19, 7101-7111.

(69) Ganjali Koli, M.; Azizi, K. The Partition and Transport Behavior of Cytotoxic Ionic Liquids (ILs) through the DPPC Bilayer: Insights from Molecular Dynamics Simulation. Mol. 
Membr. Biol. 2016, 33, 64-75.

(70) Nagle, J. F.; Zhang, R.; Tristram-Nagle, S.; Sun, W.; Petrache, H. I.; Suter, R. M. X-Ray Structure Determination of Fully Hydrated L $\alpha$ Phase Dipalmitoylphosphatidylcholine Bilayers. Biophys. J. 1996, 70, 1419-1431.

(71) Oh, K. J.; Klein, M. L. Effects of Halothane on Dimyristoylphosphatidylcholine Lipid Bilayer Structure: A Molecular Dynamics Simulation Study. Korean Chem. Soc. 2009, 30, 2087-2092.

(72) Klauda, J. B.; Venable, R. M.; Freites, J. A.; O’Connor, J. W.; Tobias, D. J.; MondragonRamirez, C.; Vorobyov, I.; MacKerell, A. D.; Pastor, R. W. Update of the CHARMM AllAtom Additive Force Field for Lipids: Validation on Six Lipid Types. J. Phys. Chem. B 2010, 114, 7830-7843.

(73) Jorgensen, W. L.; Chandrasekhar, J.; Madura, J. D.; Impey, R. W.; Klein, M. L. Comparison of Simple Potential Functions for Simulating Liquid Water. J. Chem. Phys. 1983, 79, 926935.

(74) Vanommeslaeghe, K.; Hatcher, E.; Acharya, C.; Kundu, S.; Zhong, S.; Shim, J.; Darian, E.; Guvench, O.; Lopes, P.; Vorobyov, I.; et al. CHARMM General Force Field: A Force Field for Drug-like Molecules Compatible with the CHARMM All-Atom Additive Biological Force Fields. J. Comput. Chem. 2010, 31, 671-690.

(75) Abraham, M. J.; Murtola, T.; Schulz, R.; Páll, S.; Smith, J. C.; Hess, B.; Lindah, E. Gromacs: High Performance Molecular Simulations through Multi-Level Parallelism from Laptops to Supercomputers. SoftwareX 2015, 1-2, 19-25.

(76) Humphrey, W.; Dalke, A.; Schulten, K. VMD : Visual Molecular Dynamics. J. Mol. Graph. 1996, 14, 33-38.

(77) Metso, A. J.; Jutila, A.; Mattila, J. P.; Holopainen, J. M.; Kinnunen, P. K. J. Nature of the Main Transition of Dipalmitoyphosphocholine Bilayers Inferred from Fluorescence Spectroscopy. J. Phys. Chem. B 2003, 107, 1251-1257.

(78) Suurkuusk, J.; Lentz, B. R.; Barenholz, Y.; Biltonen, R. L.; Thompson, T. E. A Calorimetric and Fluorescent Probe Study of the Gel-Liquid Crystalline Phase Transition in Small, SingleLamellar Dipalmitoylphosphatidylcholine Vesicles. Biochemistry 1976, 15, 1393-1401.

(79) Biltonen, R. L.; Lichtenberg, D. The Use of Differential Scanning Calorimetry as a Tool to Characterize Liposome Preparations. Chem. Phys. Lipids 1993, 64, 129-142.

(80) Berendsen, H. J. C.; Postma, J. P. M.; van Gunsteren, W. F.; DiNola, A.; Haak, J. R. Molecular Dynamics with Coupling to an External Bath. J. Chem. Phys. 1984, 81, 3684 3690.

(81) Essmann, U.; Perera, L.; Berkowitz, M. L.; Darden, T.; Lee, H.; Pedersen, L. G. A Smooth 
Particle Mesh Ewald Method. J. Chem. Phys. 1995, 103, 8577-8593.

(82) Hess, B. P-LINCS: A Parallel Linear Constraint Solver for Molecular Simulation. J. Chem. Theory Comput. 2008, 4, 116-122.

(83) Miyamoto, S.; Kollman, P. A. Settle: An Analytical Version of the SHAKE and RATTLE Algorithm for Rigid Water Models. J. Comput. Chem. 1992, 13, 952-962.

(84) Nosé, S. A Molecular Dynamics Method for Simulations in the Canonical Ensemble. Mol. Phys. 1984, 52, 255-268.

(85) Hoover, W. Canonical Dynamics: Equilibrium Phase-Space Distributions. Phys. Rev. A, Gen. Phys. 1985, 31, 1695-1697.

(86) Parrinello, M.; Rahman, A. Polymorphic Transitions in Single Crystals: A New Molecular Dynamics Method. J. Appl. Phys. 1981, 52, 7182-7190.

(87) Dickson, C. J.; Madej, B. D.; Skjevik, Å. A.; Betz, R. M.; Teigen, K.; Gould, I. R.; Walker, R. C.; Skjevik, A. A.; Betz, R. M.; Teigen, K.; et al. Lipid14: The Amber Lipid Force Field. J. Chem. Theory Comput. 2014, 10, 865-879.

(88) Dickson, C. J.; Rosso, L.; Betz, R. M.; Walker, R. C.; Gould, I. R. GAFFlipid: A General Amber Force Field for the Accurate Molecular Dynamics Simulation of Phospholipid. Soft Matter 2012, 8, 9617.

(89) Poger, D.; Caron, B.; Mark, A. E. Validating Lipid Force Fields against Experimental Data: Progress, Challenges and Perspectives. Biochim. Biophys. Acta-Biomembr. 2016, 1858, $1556-1565$.

(90) Saeedi, M.; Lyubartsev, A. P.; Jalili, S. Anesthetics Mechanism on a DMPC Lipid Membrane Model: Insights from Molecular Dynamics Simulations. Biophys. Chem. 2017, 226, 1-13.

(91) Granata, D.; Camilloni, C.; Vendruscolo, M.; Laio, A. Characterization of the Free-Energy Landscapes of Proteins by NMR-Guided Metadynamics. PNAS 2013, 110, 6817-6822.

(92) Minozzi, M.; Lattanzi, G.; Benz, R.; Costi, M. P.; Venturelli, A.; Carloni, P. Permeation through the Cell Membrane of a Boron-Based b -Lactamase Inhibitor. PLoS One 2011, 6, 4 9.

(93) Jambeck, J. P. M.; Lyubartsev, A. P. Exploring the Free Energy Landscape of Solutes Embedded in Lipid Bilayers. J. Phys. Chem. Lett. 2013, 4, 1781-1787.

(94) Bochicchio, D.; Panizon, E.; Ferrando, R.; Monticelli, L.; Rossi, G. Calculating the Free Energy of Transfer of Small Solutes into a Model Lipid Membrane: Comparison between Metadynamics and Umbrella Sampling. J. Chem. Phys. 2015, 143, 144108.

(95) Dama, J. F.; Parrinello, M.; Voth, G. A. Well-Tempered Metadynamics Converges Asymptotically. Phys. Rev. Lett. 2014, 112, 1-6. 
(96) Tribello, G. A.; Bonomi, M.; Branduardi, D.; Camilloni, C.; Bussi, G. PLUMED 2 : New Feathers for an Old Bird. Comput. Phys. Commun. 2014, 185, 604-613.

(97) Bonomi, M.; Branduardi, D.; Bussi, G.; Camilloni, C.; Provasi, D.; Raiteri, P.; Donadio, D.; Marinelli, F.; Pietrucci, F.; Broglia, R. A.; et al. PLUMED : A Portable Plugin for FreeEnergy Calculations with Molecular. Comput. Phys. Commun. 2009, 180, 1961-1972.

(98) Poger, D.; Mark, A. E. On the Validation of Molecular Dynamics Simulations of Saturated and Cis -Monounsaturated Phosphatidylcholine Lipid Bilayers : A Comparison with Experiment. J. Chem. Theory Comput. 2010, 6, 325-336.

(99) Leftin, A.; Molugu, T. R.; Job, C.; Beyer, K.; Brown, M. F. Area per Lipid and Cholesterol Interactions in Membranes from Separated. Biophys. J. 2014, 107, 2274-2286.

(100) King, G. I.; White, S. H. Determining Bilayer Hydrocarbon Thickness from Neutron Diffraction Measurements Using Strip-Function Models. Biophys. J. 1986, 49, 1047-1054.

(101) Kucerka, N.; Liu, Y.; Chu, N.; Petrache, H. I.; Tristram-Nagle, S.; Nagle, J. F. Structure of Fully Hydrated Fluid Phase DMPC and DLPC Lipid Bilayers Using X-Ray Scattering from Oriented Multilamellar Arrays and from Unilamellar Vesicles. Biophys. J. 2005, 88, 26262637.

(102) Shinoda, W.; Okazaki, S. A Voronoi Analysis of Lipid Area Fluctuation in a Bilayer. $J$. Chem. Phys. 1998, 109, 1517-1521.

(103) Lukat, G.; Kru, J.; Sommer, B. APL @ Voro : A Voronoi-Based Membrane Analysis Tool for GROMACS Trajectories. J. Chem. Inf. Model. 2013, 53, 2908-2925.

(104) Mori, T.; Ogushi, F.; Sugita, Y. Analysis of Lipid Surface Area in Protein - Membrane Systems Combining Voronoi Tessellation and Monte Carlo Integration Methods. J. Comput. Chem. 2011, 33, 286-293.

(105) Cheng, S. Y.; Duong, H. V; Compton, C.; Vaughn, M. W.; Nguyen, H.; Cheng, K. H. Characterization of 3D Voronoi Tessellation Nearest Neighbor Lipid Shells Provides Atomistic Lipid Disruption pro Fi Le of Protein Containing Lipid Membranes. Biophys. Chem. 2015, 198, 22-35.

(106) Exerowa, D.; Kashchiev, D. Hole-Mediated Stability and Permeability of Bilayers. Contemp. Phys. 1986, 27, 429-461.

(107) Trauble, H. The Movement of Molecules Across Lipid Membranes: A Molecular Theory. $J$. Membr. Biol. 1971, 4, 193-208.

(108) Rossum, G. Van. Python Tutorial; Technical Report CS-R9526: Amsterdam, 1995.

(109) Michaud-Agrawal, N.; Denning, J. E.; Woolf, T. B.; Beckstein, O. MDAnalysis: A Toolkit for the Analysis of Molecular Dynamics Simulations. J. Comput. Chem. 2011, 32, 23192327. 
(110) Bernal, J. D. A Geometrical Approach to the Structure Of Liquids. Nature 1959, 183, 141147.

(111) Sloan, S. W. A Fast Algorithm for Constructing Delaunay Triangulations in the Plane. Adv. Eng. Softw. 1987, 9, 34-55.

(112) Nagle, J. F. Area/Lipid of Bilayers from NMR. Biophys. J. 1993, 64, 1476-1481.

(113) Yesylevskyy, S. O.; Ramseyer, C. Determination of Mean and Gaussian Curvatures of Highly Curved Asymmetric Lipid Bilayers: The Case Study of the Influence of Cholesterol on the Membrane Shape. Phys. Chem. Chem. Phys. 2014, 16, 17052-17061.

(114) Nagle, J. F.; Tristram-Nagle, S. Structure of Lipid Bilayers. Biochim Biophys Acta. 2000, 1469, 159-195.

(115) Douliez, J.-P. P.; Léonard, A.; Dufourc, E. J.; Leonard, A. Restatement of Order Parameters in Biomembranes: Calculation of C-C Bond Order Parameters from C-D Quadrupolar Splittings. Biophys J 1995, 68, 1727-1739.

(116) Doktorova, M.; Levine, M. V; Khelashvili, G.; Weinstein, H. A New Computational Method for Membrane Compressibility : Bilayer Mechanical Thickness Revisited. Biophys. J. 2019, $116,1-16$.

(117) Comer, J.; Schulten, K.; Chipot, C. Permeability of a Fluid Lipid Bilayer to Short-Chain Alcohols from First Principles. J. Chem. Theory Comput. 2017, 13, 2523-2532.

(118) Lee, B. L.; Kuczera, K. Simulating the Free Energy of Passive Membrane Permeation for Small Molecules. Mol. Simul. 2018, 44, 1147-1157.

(119) Kumari, P.; Kaur, S.; Sharma, S.; Kashyap, H. K. Impact of Amphiphilic Molecules on the Structure and Stability of Homogeneous Sphingomyelin Bilayer: Insights from Atomistic Simulations. J. Chem. Phys. 2018, 148, 165102.

(120) Shinoda, W. Permeability across Lipid Membranes. Biochim. Biophys. Acta-Biomembr. 2016, 1858, 2254-2265. 
Table of Contents Graphics:

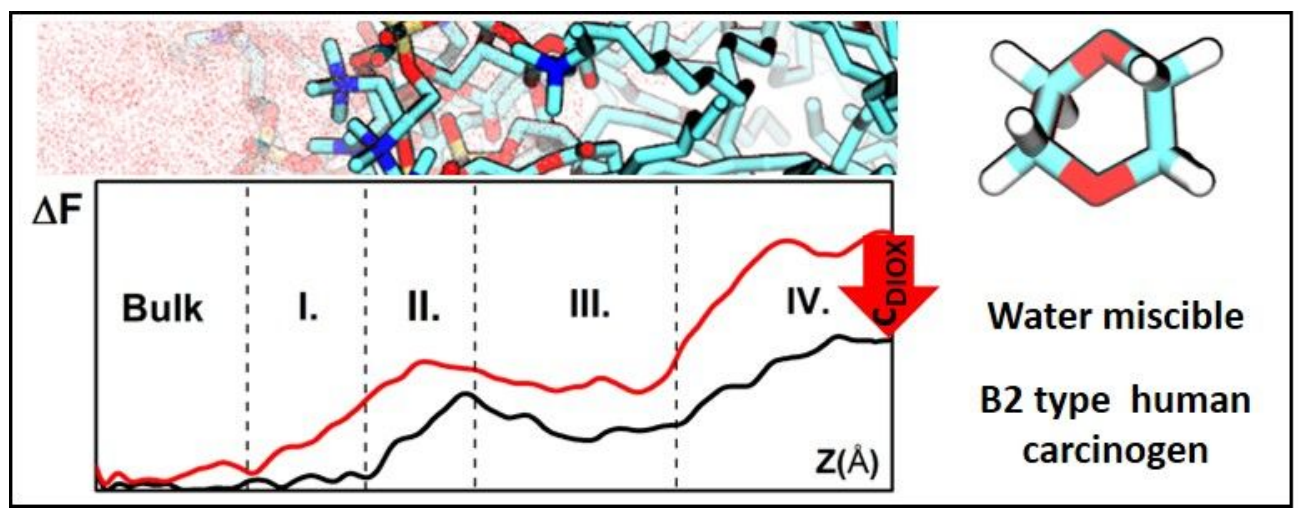

